\title{
Insight into performance of quantum dot infrared photodetectors
}

\author{
P. MARTYNIUK and A. ROGALSKI* \\ Institute of Applied Physics, Military University of Technology, 2 Kaliskiego Str., 00-908 Warsaw, Poland
}

\begin{abstract}
In the paper, an algorithm for theoretical evaluation of dark and illumination characteristics of quantum dot infrared photodetectors (QDIPs) is presented. The developed algorithm is based on a model previously published by Ryzhii and co-workers. In our considerations it is assumed that both thermionic emission and field-assisted tunnelling mechanisms determine the dark current of quantum dot detectors. The model permits to calculate the dark current, current gain, average number of electrons in quantum dots, photocurrent, and detector responsivity as a function of the structural parameters. Moreover, it explains some features of QDIP characteristics.

In several cases, the theoretical predictions are compared with experimental data. Good agreement between both kinds of data has been obtained.
\end{abstract}

Key words: QDIPs, dark current, current gain, responsivity, detectivity.

\section{Introduction}

The most advanced III-V infrared detectors, which utilize intersubband or subband to continuum transitions in quantum wells, are GaAs/AlGaAs quantum well infrared photodetectors (QWIPs). The imaging performance of focal plane arrays fabricated with this material system is comparable to the state of art of $\mathrm{HgCdTe}[1,2]$. The success of quantum well structures for infrared detection applications has stimulated the development of quantum dot infrared photodetectors (QDIPs). In the past decade, the QDIPs have become a topic of extensive research not only for the fundamental understanding of fascinating physics that exists in zero-dimensional systems but also for their application in infrared optoelectronics [35]. At present, nearly defect-free quantum dot devices can be fabricated reliably and reproducibly.

In general, QDIPs are similar to QWIPs but with the quantum wells replaced by quantum dots, which have size confinement in all spatial directions. Recently, Krisha et al. [6] has reviewed progress of QD-based focal plane arrays (FPAs) from the first raster scanned image to a $640 \times 512$ camera. Also first two-color QDIP camera has been demonstrated [7].

In this paper we improve the theoretical model of quantum dot detector elaborated by Ryzhii and co-workers [8-10] to describe the behaviour of QDIPs under dark and illuminated conditions. We use this model to estimate influence of some structural device parameters (geometry, impurity doping, density of dots, number of QD layers), bias voltage and operation temperature on detector performance. In several cases, the theoretical predictions are compared with experimental data.

\section{Anticipated advantages of QDIPs}

The quantum-mechanical nature of QDIPs leads to several advantages over QWIPs and other types of IR detectors that are available. As in the $\mathrm{HgCdTe}$, QWIP and type II superllat- ice technologies, QDIPs provide multi-wavelength detection. However, QDs provide many additional parameters for tuning the energy spacing between energy levels, such as QD size and shape, strain, and material composition.

The potential advantages in using QDIPs over quantum wells are as follows:

- Intersubband absorption may be allowed at normal incidence (for n-type material). In QWIPs, only transitions polarized perpendicularly to the growth direction are allowed, due to absorption selection rules. The selection rules in QDIPs are inherently different, and normal incidence absorption is observed.

- Thermal generation of electrons is significantly reduced due to the energy quantization in all three dimensions. As a result, the electron relaxation time from excited states increases due to phonon bottleneck. Generation by LO phonons is prohibited unless the gap between the discrete energy levels equals exactly to that of the phonon. This prohibition does not apply to quantum wells, since the levels are quantized only in the growth direction and a continuum exists in the other two directions (hence generationrecombination by LO phonons with capture time of a few picoseconds). Thus, it is expected that $\mathrm{S} / \mathrm{N}$ ratio in QDIPs will be significantly larger than that of QWIPs.

- Lower dark current of QDIPs is expected than of $\mathrm{HgCdTe}$ detectors and QWIPs due to 3-D quantum confinement of the electron wavefunction.

Both the increased electron lifetime and the reduced dark current indicate that QDIPs should be able to provide high temperature operation. In practice, however, it has been a challenge to meet all of above expectations.

Carrier relaxation times in QDs are longer than the typical 1-10 ps measured for quantum wells. It is predicted that the carrier relaxation time in QDs is limited by electron-hole scattering [11], rather than phonon scattering. For QDIPs, the life-

*e-mail: rogan@wat.edu.pl 
time is expected to be even larger, greater than $1 \mathrm{~ns}$, since the QDIPs are majority carrier devices due to absence of holes.

The main disadvantage of the QDIP is the large inhomogeneous linewidth of the quantum-dot ensemble variation of dot size in the Stranski-Krastanow growth mode [12]. As a result, the absorption coefficient is reduced, since it is inversely proportional to the ensemble linewidth. Large, inhomogeneously broadened linewidth has a deleterious effect on QDIP performance. Subsequently, the quantum efficiency QD devices tend to be lower than what is predicted theoretically. Vertical coupling of quantum-dot layers reduces the inhomogeneous linewidth of the quantum-dot ensemble; however, it may also increase the dark current of the device, since carriers can tunnel through adjacent dot layers more easily. As in other type of detectors, also nonuniform dopant incorporation adversely affects the performance of the QDIP. Therefore, improving QD uniformity is a key issue in the increasing absorption coefficient and improving the performance. Thus, the growth and design of unique QD heterostructure is one of the most important issues related to achievement of state-of-the art QDIP performance.

\section{QDIP model}

In further considerations a QDIP model developed by Ryzhii et al. is adapted [8-10]. The QDIP consists of a stack of QD layers separated by wide-gap material layers (see Fig. 1). Each QD layer includes periodically distributed identical QDs with the density $\Sigma_{Q D}$ and sheet density of doping donors equal to $\Sigma_{D}$. In the realistic QDIPs, the lateral size of QDs, $a_{Q D}$, is sufficiently large in comparison with the transverse size, $h_{Q D}$. Consequently, only two energy levels associated with the quantization in the transverse direction exist. Relatively sufficiently large lateral size, $l_{Q D}$, causes a large number of bound states in dots and, consequently, is capable to accepting large number of electrons. Instead, the transverse size is small in comparison with the spacing between the QD layers, $L$. The lateral spacing between QDs is equal to $L_{Q D}=\sqrt{\Sigma_{Q D}}$. The average number of electrons in a QD belonging to the $k$-th QD layer, $\left\langle N_{k}\right\rangle$, can be indicated by a solitary QD layer in- $\operatorname{dex}(k=1,2, \ldots, K$, where $K$ is the total number of the QD layers). The QDIP active region (the stack of QD arrays) is sandwiched between two heavily doped regions which serve as the emitter and collector contacts.

Generally, in theoretical considerations it is assumed that the dark current across the device resulting only from carrier trapping into and thermionic emission from the QDs. However, experimental measurements of dark current indicate on considerable contribution of the field-assisted tunnelling through the triangular potential barrier, $\phi_{B}$, particularly at higher operating temperature and larger applied voltage. In this paper, a model which includes both thermionic emission and field-assisted tunnelling is used.

Figure 1(b) illustrates schematically quantum dot capture, thermionic emission and field-assisted tunnelling processes considered in the detailed balance relation under dark conditions. Both in dark and under illumination, the current across the QDIP at the applied voltage is accompanied by several processes such as: thermo- and photoexcitation of electrons from bound states in QDs into continuum states, capture of mobile electrons into QDs, transport of electrons between the charged QDs, and injection of extra electrons from the emitter contact. The last process is caused by redistribution of the potential in the detector active region as a result of change in the charges accumulated by QDs, and collection of the excited and injection electrons by the pertinent contact [9].

According to Ref. 8, the average dark current density in a QDIP can be expressed as

$$
\left\langle j_{\text {dark }}\right\rangle=j_{\max } \Sigma_{Q D} \int_{0}^{\infty} d r^{2} \exp \left(\frac{q \varphi\left(\left\langle N_{k}\right\rangle\right)}{k T}\right),
$$

where $\varphi\left(\left\langle N_{k}\right\rangle\right)$ is the potential distribution in the quantum dot layer as a function of the average number of electrons in each QD, and $j_{\max }$ is the maximum current density provided by the top $n^{+}$-contact. The value of this current can be estimated from Richardson-Dushman relation

$$
j_{\max }=A^{*} T^{2}
$$

where $A^{*}$ is the Richardson's constant.

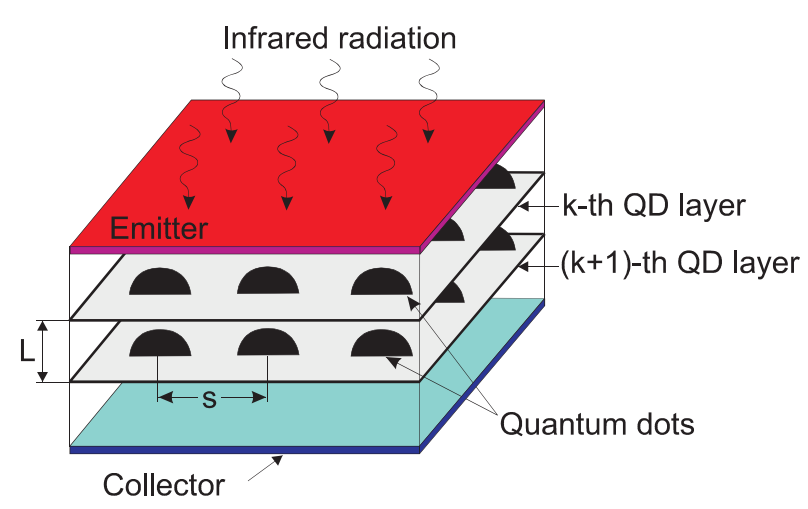

(a)

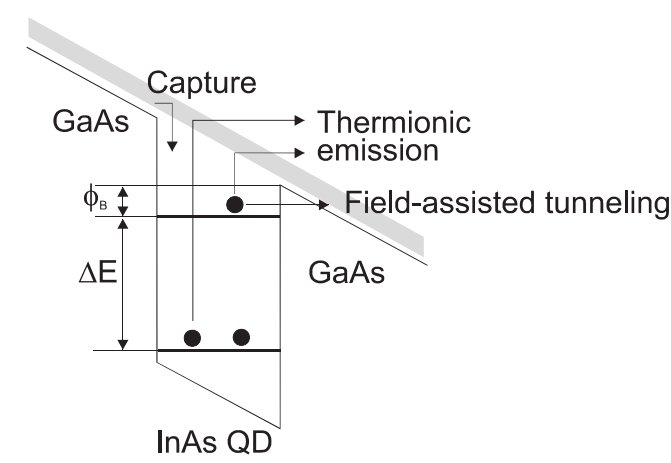

(b)

Fig. 1. Schematic view of the quantum dot structure (a) and conduction band structure of the dot (b) 
It can show that

$$
\left\langle j_{\text {dark }}\right\rangle=j_{\max } \frac{\Theta}{\langle N\rangle} \exp \left[q \frac{V+V_{D}-\left(\langle N\rangle / N_{Q D}\right) V_{Q D}}{(K+1) k T}\right] \text {, }
$$

where

$$
\Theta=\frac{\pi}{4}\left[\operatorname{erf}\left(0.47 L_{Q D} \sqrt{\frac{\langle N\rangle \Sigma_{Q D}^{3 / 2}}{\varepsilon_{o} \varepsilon_{r} k_{B} T}}\right)\right]^{2} \frac{\varepsilon_{o} \varepsilon_{r} k_{B} T}{q^{2} \sqrt{\Sigma_{Q D}}},
$$

and

$$
\begin{gathered}
V_{Q D}=\frac{q}{2 \varepsilon_{o} \varepsilon_{r}} K(K+1) \Sigma_{Q D} L(1-\vartheta) N_{Q D}, \\
V_{D}=\frac{q}{2 \varepsilon_{o} \varepsilon_{r}} K(K+1) \Sigma_{D} L
\end{gathered}
$$

are the characteristic voltages. In the above equations, $V$ is the applied voltage, $q$ is the electron charge, $\varepsilon_{r}$ is the dielectric constant of the material from which the QD is fabricated, but

$$
\vartheta=\frac{0.72 \sqrt{2}}{\pi K L \sqrt{\Sigma_{Q D}}} .
$$

The value of $\left\langle N_{k}\right\rangle$ can be derived from a detailed balance relation accounting for emission and capture at a QD. The balance equation to equate the rates of electron capture into and emission from QDs under dark conditions is

$$
\left\langle j_{\text {dark }}\right\rangle=\frac{q \Sigma_{Q D}}{p_{k}}\left(G_{t h}+G_{\text {tun }}\right),
$$

where $p_{k}$ is the capture probability, $G_{\text {th }}$ is the rate of thermionic emission, and $G_{\text {tun }}$ is the rate of field-assisted tunnelling emission. The capture probability and thermionic emission rate have been discussed previously in detail [8]. The expressions for these terms are

$$
p_{k}=p_{o k} \frac{N_{Q D}-\left\langle N_{k}\right\rangle}{N_{Q D}} \exp \left[-\frac{q^{2}\left\langle N_{k}\right\rangle}{C_{Q D} k T}\right]
$$

and

$$
G_{\text {th }}=G_{o} \exp \left(-\frac{E_{Q D}}{k T}\right) \exp \left[\frac{\pi \eta^{2}\left\langle N_{k}\right\rangle}{m^{*} k T a_{Q D}^{2}}\right],
$$

where $p_{O}$ is the capture probability for uncharged QDs close to $1, N_{Q D}$ is the maximum number of electrons which can occupy each QD, $G_{O}$ is the thermionic emission rate constant (a factor that depends on a detector structure), $E_{Q D}$ is the ionization energy of the ground state in QDs, $m^{*}$ is the effective mass of the electron, $\eta$ and $k$ are the Planck and Boltzmann constants, respectively, and $T$ is the temperature. $C_{Q D}$ is the QD capacitance given by

$$
C=\frac{8 \varepsilon_{o} \varepsilon_{r} a_{Q D}}{\sqrt{\pi}} .
$$

Using the Wentzel-Kramer-Brillouin approximation for one-dimensional triangular barrier, the field-assisted tunnelling rate is expressed by

$$
\begin{aligned}
& G_{\text {tu } n}=G_{o t} \exp \\
&\left(-\frac{4}{3} \frac{\sqrt{2 m^{*} q}}{\eta} \frac{\phi_{B}^{3 / 2}}{E}\right) \exp \left(-\frac{\Delta E}{k T}\right) \\
& \times \exp \left[\frac{\pi \eta^{2}\left\langle N_{k}\right\rangle}{m^{*} k T a_{Q D}^{2}}\right],
\end{aligned}
$$

where $G_{o t}$ is the field-assisted tunnelling emission rate constant, and $E$ is the electric field across the device.

The potential barrier height is expressed by

$$
\phi_{B}=\frac{E_{Q D}-\Delta E}{q} .
$$

The quantities in expressions (9), (10), and (12) can be calculated explicitly, or determined experimentally, except for $G_{o}, G_{o t}$, and $\Delta E$. In this paper, these parameters are used as fitting parameters in the model and are based on theoretical estimations and experimental data provided by Stiff-Roberts et al. [13].

Figure 2 shows the temperature dependence of $G_{o}, G_{o t}$, and $j_{\text {max }}$. As we can see, $G_{o}$ is almost unchanged with increasing temperature, as expected and its value has been estimated as $10^{10} \mathrm{~s}^{-1}$. However, $G_{o t}$ demonstrates a significant decrease with temperature increasing. It indicates that at low temperature, the tunnelling current has considerable contribution in dark current value. Sequential resonant tunnelling, which has been observed in quantum-well infrared photodetectors [14], could explain the observed behaviour.

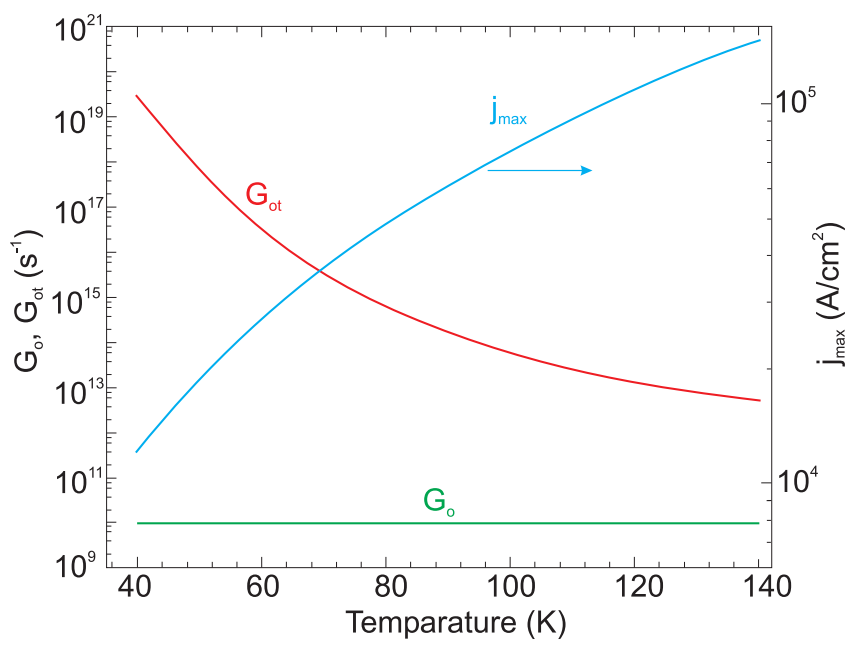

Fig. 2. Temperature dependence of fitting parameters of $G_{o}, G_{o t}$, and $j_{\max }$

An interesting feature that characterizes the QDIPs is the existence of current gain. The gain can exhibit a broad range of values depending on the bias and the type of material investigated. Assuming that the capture probability is small and the transit time across one period, $\tau_{\text {trans }}$, is considerably smaller than the recombination time from an extended state back into a $\mathrm{QD}, \tau_{\text {life }}$, we have

$$
g=\frac{1}{K} \frac{\tau_{\text {life }}}{\tau_{\text {trans }}}=\frac{1}{K} \frac{1}{\tau_{\text {trans }} / \tau_{\text {life }}}=\frac{1}{K p_{o k}},
$$

where $p_{o k}$ is the neutral capture probability [see Eq. (9)] and is equal to the ratio of the transit time, $\tau_{\text {trans }}$, and the capture time, $\tau_{\text {life, }}$,

$$
p_{o k}=\frac{\tau_{\text {trans }}}{\tau_{\text {life }}} .
$$

The current gain is determined by the capture probability and the number of QD layers. The product $K \tau_{\text {trans }}$ is the total transit time of the device. 
Table 1

Theoretically calculated quantum dot's values of electron mobility, transit time and exponential term of Eq. (9) in dependence of trap energy and temperature

\begin{tabular}{|c|c|c|c|c|c|c|c|}
\hline \multirow{3}{*}{$\mathrm{T}[\mathrm{K}]$} & \multicolumn{3}{|c|}{$\mu\left[\mathrm{cm}^{2} / \mathrm{Vs}\right]$} & \multicolumn{3}{|c|}{$\tau_{\text {trans }}[\mathrm{s}]$} & \multirow{3}{*}{$\exp \left[-q^{2}\left\langle N_{k}\right\rangle / C_{Q D} k_{B} T\right]\left\langle N_{k}\right\rangle=0.5$} \\
\hline & \multicolumn{3}{|c|}{$E_{t}[\mathrm{eV}]$} & \multicolumn{3}{|c|}{$E_{t}[\mathrm{eV}]$} & \\
\hline & 0.02 & 0.06 & 0.07 & 0.02 & 0.06 & 0.07 & \\
\hline 60 & $9.11 \times 10^{2}$ & $1.8 \times 10^{0}$ & $2.10 \times 10^{-1}$ & $7.83 \times 10^{-14}$ & $3.09 \times 10^{-11}$ & $2.63 \times 10^{-10}$ & 0.11 \\
\hline 80 & $9.43 \times 10^{2}$ & $2.1 \times 10^{1}$ & $4.43 \times 10^{0}$ & $7.68 \times 10^{-14}$ & $2.57 \times 10^{-12}$ & $1.24 \times 10^{-11}$ & 0.19 \\
\hline 100 & $9.58 \times 10^{2}$ & $9.5 \times 10^{1}$ & $2.82 \times 10^{1}$ & $7.61 \times 10^{-14}$ & $5.83 \times 10^{-13}$ & $1.94 \times 10^{-12}$ & 0.27 \\
\hline 120 & $9.66 \times 10^{2}$ & $2.2 \times 10^{2}$ & $9.33 \times 10^{1}$ & $7.57 \times 10^{-14}$ & $2.42 \times 10^{-13}$ & $5.91 \times 10^{-13}$ & 0.34 \\
\hline 140 & $9.71 \times 10^{2}$ & $3.9 \times 10^{2}$ & $2.03 \times 10^{2}$ & $7.55 \times 10^{-14}$ & $1.48 \times 10^{-13}$ & $2.74 \times 10^{-13}$ & 0.39 \\
\hline 160 & $9.75 \times 10^{2}$ & $5.3 \times 10^{2}$ & $3.35 \times 10^{2}$ & $7.53 \times 10^{-14}$ & $1.14 \times 10^{-13}$ & $1.71 \times 10^{-13}$ & 0.44 \\
\hline 180 & $9.77 \times 10^{2}$ & $6.4 \times 10^{2}$ & $4.62 \times 10^{2}$ & $7.52 \times 10^{-14}$ & $9.90 \times 10^{-14}$ & $1.28 \times 10^{-13}$ & 0.48 \\
\hline 200 & $9.79 \times 10^{2}$ & $7.5 \times 10^{2}$ & $5.69 \times 10^{2}$ & $7.51 \times 10^{-14}$ & $9.12 \times 10^{-14}$ & $1.08 \times 10^{-13}$ & 0.52 \\
\hline
\end{tabular}

Table 2

Typical parameter values of QDIP fabricated from GaAs or InGaAs

\begin{tabular}{ccccccc}
\hline \hline$a_{Q D}$ & $h$ & $\Sigma_{Q D}$ & $\Sigma_{D}$ & $L$ & $K$ & $N_{Q D}$ \\
\hline $10-40 \mathrm{~nm}$ & $4-8 \mathrm{~nm}$ & $(1-10) 10^{10} \mathrm{~cm}^{-2}$ & $(0.3-0.6) \Sigma_{Q D}$ & $40-100 \mathrm{~nm}$ & $10-70$ & 8 \\
\hline
\end{tabular}

To estimate both $\tau_{\text {life }}$ and $\tau_{\text {trans }}$ times, we follow Ref. 15 We will treat the capture rate, $V_{t}$, as a parameter not to tray to study its intricate and subtle structure. Then,

$$
\tau_{\text {life }}=\frac{(K+1) L}{\pi a_{Q D}^{2} h_{Q D} \Sigma_{Q D} V_{t}} .
$$

The transit time across one period of QD layer is rather complicated function of the electric field, $E$, the carrier mobility, $\mu$, the effective trap energy, $E_{t}$, and the volume concentration of traps. In general it can be assumed that

$$
\tau_{\text {trans }}=\frac{h_{Q D}}{\mu E\left[1+\left(\mu E / v_{s}\right)^{2}\right]^{-1 / 2}} .
$$

Table 1 contains the results of calculations of electron mobility, transit time and exponential term of Eq. (9) in dependence on the trap energy $E_{t}=0.02,0.06,0.07 \mathrm{eV}$ and the temperature $T=60-200 \mathrm{~K}$. According to Ref. 15, the expected values of trap energies are in the range from 0.092 to $0.06 \mathrm{eV}$.

Using equations (3) and (8) we have

$$
\begin{gathered}
j_{\max } \frac{\Theta}{\langle N\rangle} \exp \left[q \frac{V+V_{D}-\left(\langle N\rangle / N_{Q D}\right) V_{Q D}}{(K+1) k T}\right] \\
=K q \Sigma_{Q D} G_{\text {th }} g+K q \Sigma_{Q D} G_{\text {tun }} g .
\end{gathered}
$$

Solving numerically this equation, we can determine the self-consistent average number of electrons in QDs $\langle N\rangle$ and next other detector's parameters.

Table 2 contains the reference values of QD parameters. These values are considered for a QDIP fabricated from
GaAs or InGaAs. The self-assembled dots formed by epitaxial growth are typically pyramidal to lens shaped with a base dimension of 10-20 nm and height of 4-8 nm with an areal density determined to be $5 \times 10^{10} \mathrm{~cm}^{-2}$ using atomic force microscopy.

\section{Results and discussion}

In the next two sections of the paper, we give some numerical results for the interesting QDIP parameter values to explain the behaviour of detector under the variation of these parameters. Part of theoretical predictions is compared with experimental data published in literature. Material parameters that are used for QDIP calculations are listed in Table 3. They are representative for self-assembled InAs/GaAs quantum dots reported in the literature $[3-5,12,13]$.

In several cases, the theoretical predictions will be compared with experimental data reported in Ref. 16 for InAs/GaAs QDs grown by molecular beam epitaxy on semiinsulating $\operatorname{GaAs}(100)$ substrates. The QDs layers with a dot density of $10^{10}-10^{11}$ dots $/ \mathrm{cm}^{2}$ were silicon-doped to a level of $n=(0.5-1) \times 10^{18} \mathrm{~cm}^{-3}$. The doping level corresponds to $\sim 0.5-1$ dopant atoms/dot. Cross-section transmission electron microscope images indicate that the dots were similar to a lens in shape with a diameter of $\sim 15 \mathrm{~nm}$ and a height of $\sim 5 \mathrm{~nm}$. The active region of circular mesa QDIP structures (400 $\mu \mathrm{m}$ in diameter) consisted of ten layers of Si-doped QDs with 100-nm undoped GaAs barriers sandwiched between $\mathrm{n}^{+}$-

\begin{tabular}{|c|c|c|c|c|c|c|c|c|}
\hline$a_{Q D}$ & $\Sigma_{Q D}$ & $\Sigma_{D}$ & $L$ & $K$ & $N_{Q D}$ & $A$ & $\varepsilon_{r}$ & $m^{*} / m$ \\
\hline $15 \mathrm{~nm}$ & $10^{11} \mathrm{~cm}^{-2}$ & $(1-10) \times 10^{10} \mathrm{~cm}^{-2}$ & $40-100 \mathrm{~nm}$ & $10-100$ & 6 & $200 \times 200 \mu \mathrm{m}^{2}$ & 12 & 0.023 \\
\hline
\end{tabular}
GaAs contact layers.

Table 3

Material parameters of quantum dots in QDIP calculations 
Absorption measurements of InAs/GaAs QDIPs demonstrated tunable operating wavelengths between $6-18 \mu \mathrm{m}$ using subband-subband or subband-continuum transitions. The QD absorption spectrum is generally broad due to the size variation of QDs comprising the active region of a device. Generally, the tradeoff between the absorption coefficient and the absorption linewidth is observed. Thus, even though each QD individually demonstrates sharp atom-like states, for an array of QDs the density of states is an inhomogeneous Gaussian distribution. The optical absorption between the ground and excited levels is found to have a value [17]

$$
\alpha \approx \frac{3.5 \times 10^{5}}{\sigma}, \quad\left[\text { in } \mathrm{cm}^{-1}\right]
$$

which is inversely proportional to the linewith of the transition in $\mathrm{meV}$.

In our paper, some theoretical predictions are compared with experimental data [16] for the samples characterized by broad absorption near $8 \mu \mathrm{m}\left(E_{Q D}=155 \mathrm{meV}\right.$ at $\left.40 \mathrm{~K}\right)$ which is in the range of the energy separation between the quantum dot ground state and the GaAs continuum.

\subsection{QDIP's characteristics in dark conditions. Dark cur-} rent. The dark current is an important parameter in photodetector characterization because it determines the base current level that photocurrent must exceed in order to be detected. In our considerations we assume that possible sources of dark current in QDIP include thermal generation of carriers, thermionic emission from QDs, and field-assisted tunnelling emission from QDs (other sources, e.g. impact-ionization in the active region, surface leakage current and ohmic leakage current are omitted).

Figures 3-6 give the change of the dark current with applied voltage, temperature, doping sheet density, number of QD layers and spacing between QD layers. The theoretical dark current-voltage characteristics in the temperature range between 40 and $80 \mathrm{~K}$ are shown in Fig. 3. As expected, the dark current increases with temperature increasing. This figure demonstrates also excellent agreement between theoretical predictions and experimental data for similar detector structure at $40 \mathrm{~K}$ [16].

An additional insight in I-V characteristic gives Fig. 4, where influence of the potential barrier height, $\phi_{B}$, on dark current is presented. It is clearly shown that $\phi_{B}$ affect the dark current, but mainly in the region of low bias voltage. Decreasing barrier height causes increasing dark current.

In general, the dark current depends on bias voltage and operation temperature and it is determined by a generationrecombination processes in a detector active region. Influence of these processes depends on electrical charge gathered in QDs and on trapping energy. Changes of electron mobility and transit time in dependence on trap energy are shown in Table 1.

The number of electrons inside the QDs is determined by the balance of the trapped current into the QDs with the emitted current from the QDs. When the current increases with temperature and voltage, the current number inside the
QD increases and the Fermii level also increases. As a result, the capture probability decreases and the current gain shows a dramatic increase with temperature and bias.

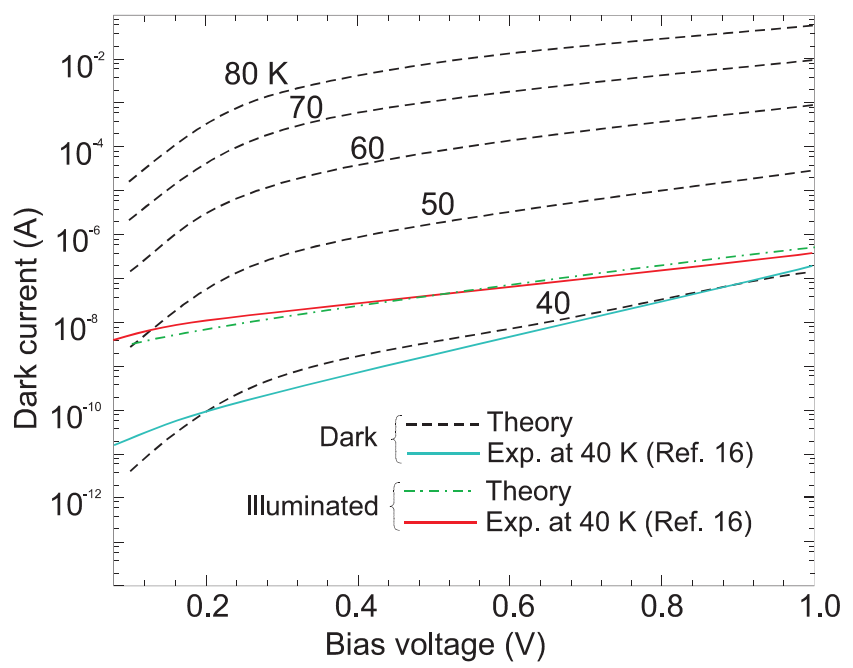

Fig. 3. Current-voltage characteristics versus applied voltage at different temperatures. In theoretical estimation, the following parameters have been assumed: $a_{Q D}=15 \mathrm{~nm}, \Sigma_{Q D}=10^{11} \mathrm{~cm}^{-2}, \Sigma_{D}=$ $5 \times 10^{10} \mathrm{~cm}^{-2}, K=10, L=100 \mathrm{~nm}, N_{Q D}=6, \phi_{B}=0.005 \mathrm{eV}$, and incident photon flux $\Phi_{s}=8 \times 10^{17}$ photons $/ \mathrm{cm}^{2} \mathrm{~s}$ for QDIP at $40 \mathrm{~K}$. The experimental data (solid lines) at $40 \mathrm{~K}$ are after Ref. 16.

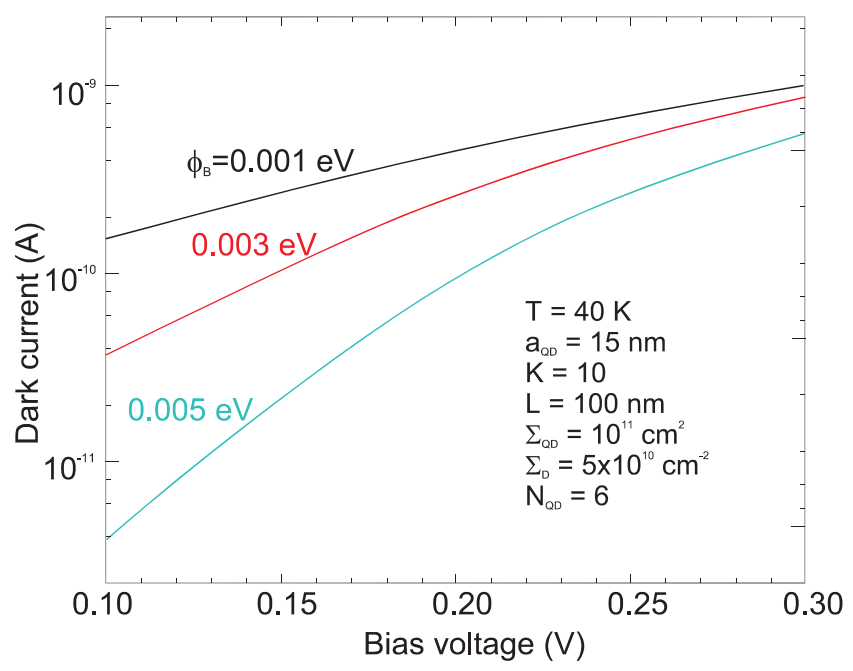

Fig. 4. Influence of the potential barrier height, $\phi_{B}$, on I-V characteristics at $40 \mathrm{~K}$. In theoretical estimation, the following parameters have been assumed: $a_{Q D}=15 \mathrm{~nm}, \Sigma_{Q D}=10^{11} \mathrm{~cm}^{-2}$, $\Sigma_{D}=5 \times 10^{10} \mathrm{~cm}^{-2}, K=10, L=100 \mathrm{~nm}, N_{Q D}=6$, and $\phi_{B}=0.001-0.005 \mathrm{eV}$.

Figure 5 presents the dependence of dark current on the density of quantum dots and doping sheet density of QDs. At constant doping density $\Sigma_{D}=5 \times 10^{10} \mathrm{~cm}^{-2}$ [see Fig. 5(a)], the dark current decreases with increasing the QD density due to decreasing number of carriers in quantum dots. The decrease in repulsive potential of charge carriers in quantum dots causes increase in the electron capture probability and decrease in the current gain. In the range of high QD density, the dark current saturates on different levels in depen- 
dence on a bias voltage. However, as expected, with increasing value of the sheet density at constant QD density equal to $\Sigma_{Q D}=10^{11} \mathrm{~cm}^{-2}$, the value of dark current increases [Fig. 5(b)].

a)

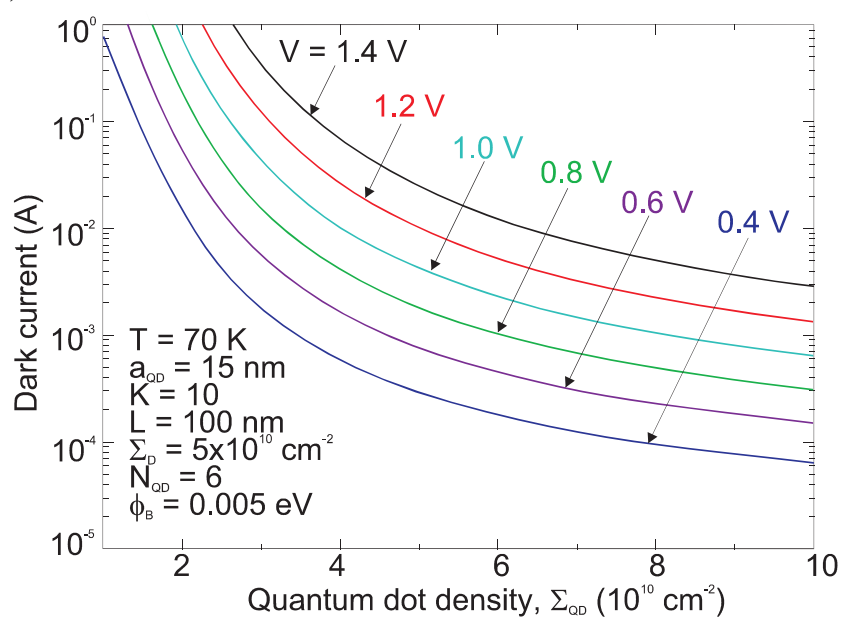

b)

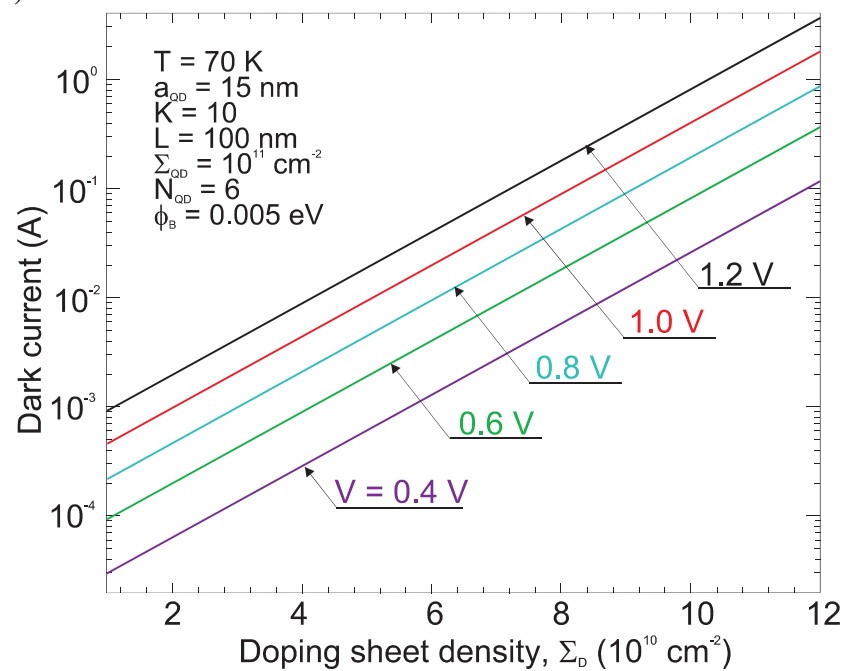

Fig. 5. Dark current versus the quantum dot density, $\Sigma_{Q D}$ (a) and the doping sheet density, $\Sigma_{D}$ (b) for different bias voltages at temperature $70 \mathrm{~K}$. In theoretical estimation, the following parameters have been assumed: $a_{Q D}=15 \mathrm{~nm}, \Sigma_{Q D}=10^{11} \mathrm{~cm}^{-2}, K=10$, $L=100 \mathrm{~nm}, N_{Q D}=6$, and $\phi_{B}=0.005 \mathrm{eV}$.

Next figure (Fig. 6) depicts the change of dark current with a number of quantum dot layers at different spacings between quantum dot layers (a) and the change with spacing between quantum dot layers at different bias voltages (b). Parameters taken in the calculations are marked inside the figures. We can see that increasing number of quantum dot layers (spacing between QD layers) causes decreasing of the dark current. In the range of a number of QD layers $K<20$, strong increase in dark current is observed. Increasing spacing between QD layers from $50 \mathrm{~nm}$ to $110 \mathrm{~nm}$ results in decreasing dark current more than one order of magnitude.

Influence of bias voltage on a dark current is stronger, what is shown in Fig. 6(b). Changes of bias voltage, from
$0.4 \mathrm{~V}$ to $1.2 \mathrm{~V}$, results in increasing the dark current approximately of about two orders of magnitude almost independently on spacing between QD layers.

a)

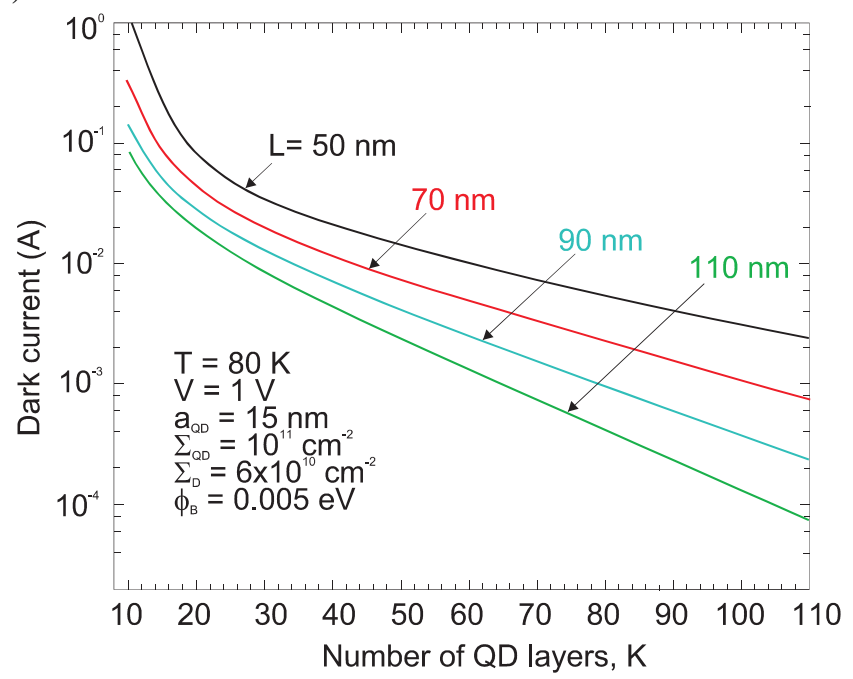

b)

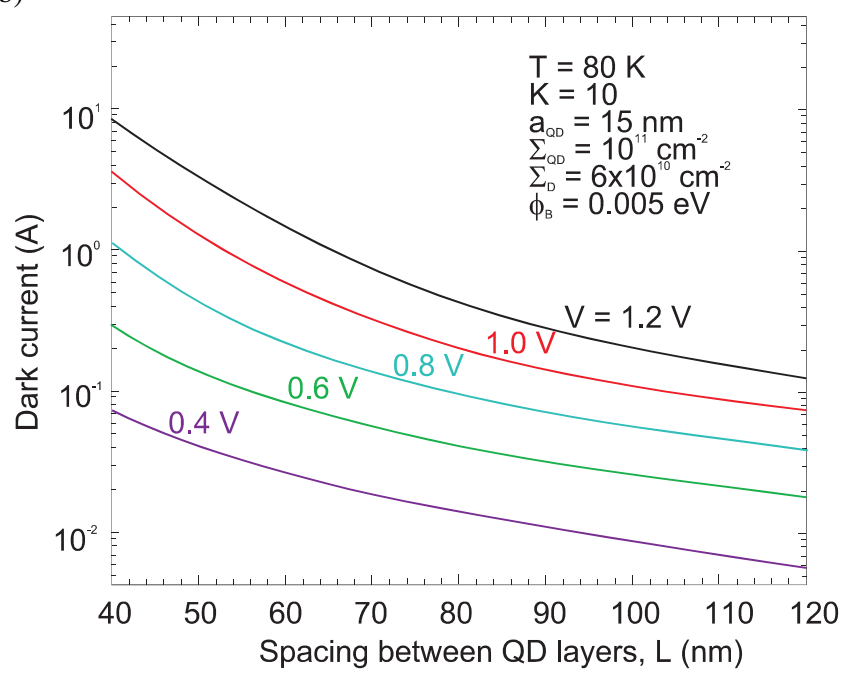

Fig. 6. Dark current versus a number of quantum dot layers, $K$ (a) and spacing between quantum dot layers, $L$ (b). Calculations have been performed for detector parameters shown inside the figures.

Current gain. Current gain of QDIPs has been reported by many research groups and the reported values span a very broad range form 1 to $10^{6}$ depending upon the material, applied bias, and temperature [18-20]. Recently published theory of diffusion and recombination in QDIPs explains the reported values of gain [15]. Results of Ref. 15 are adapted for our calculations.

Figure 7 presents dependence of the current gain on temperature and bias voltage. The current gain increases more than 100 times from $60 \mathrm{~K}$ to $140 \mathrm{~K}$. The increasing dark current with the temperature injects more carriers inside the QDs. The carrier capture probability through the QD layer changed dramatically within the temperature range. The capture process is influenced by the repulsive Coulomb potential of the extra carriers inside the quantum 
dots. The repulsive potential of the extra carriers suppresses the capture process and enhances the current gain. Unlike in the quantum wells, where carriers flow freely in the $x-y$ direction, the isolated charge in the QD could generate potential barrier to electrons passing through the QD layers.

The current gain increases with the bias voltage, what has been confirmed experimentally. Good agreement between theoretical predictions and experimental data is shown in Fig. 7(b) for QDIP operating at $40 \mathrm{~K}$.

a)

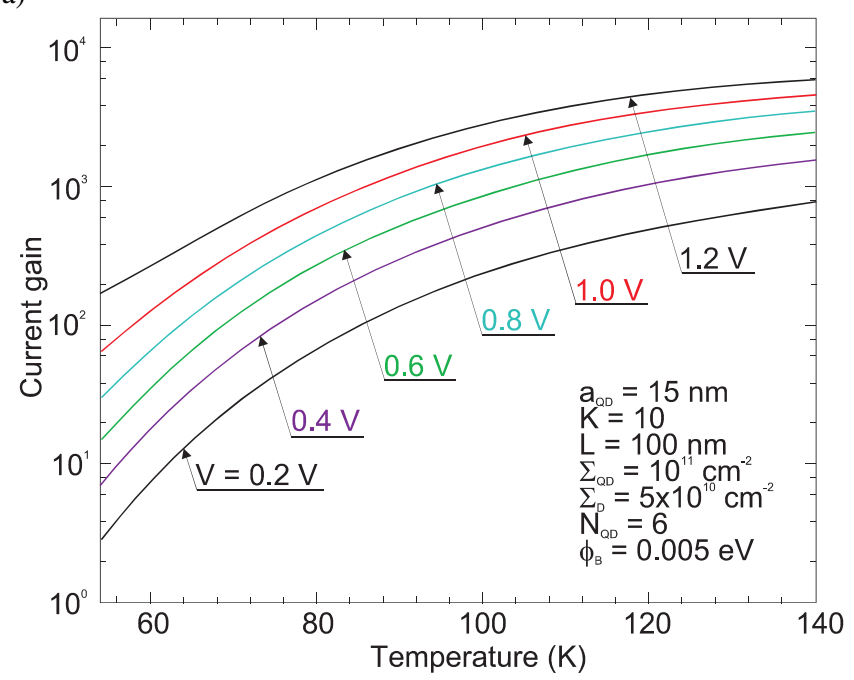

b)

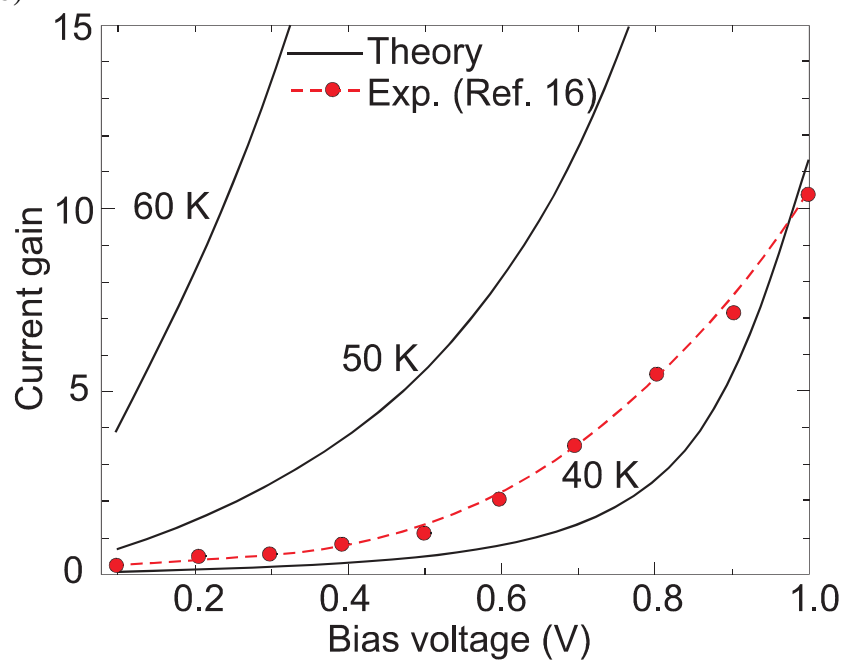

Fig. 7. Current gain as a function of temperature (a) and bias voltage (b). In theoretical estimation, the following parameters have been assumed: $a_{Q D}=15 \mathrm{~nm}, \Sigma_{Q D}=10^{11} \mathrm{~cm}^{-2}, \Sigma_{D}=5 \times 10^{10} \mathrm{~cm}^{-2}$, $K=10, L=100 \mathrm{~nm}, N_{Q D}=6$, and $\phi_{B}=0.005 \mathrm{eV}$. The experimental data at $40 \mathrm{~K}$ are after Ref. 16.

The current gain indeed exhibits a broad range of values, including up to several thousands, depending on the type of material investigated (especially density of quantum dots and doping density) and bias. These dependences are shown in Fig. 8. We can notice that the dependence of current gain on QD density is similar to dependence of responsivity on $\Sigma_{Q D}$ [see Fig. 5(a)]. This shows that the change of responsivity is dominated by the current gain.

a)

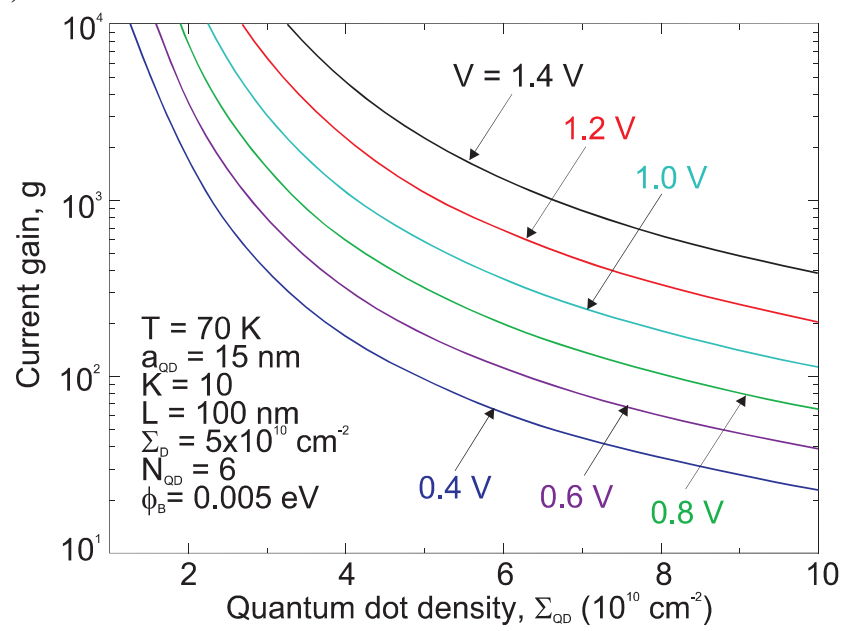

b)

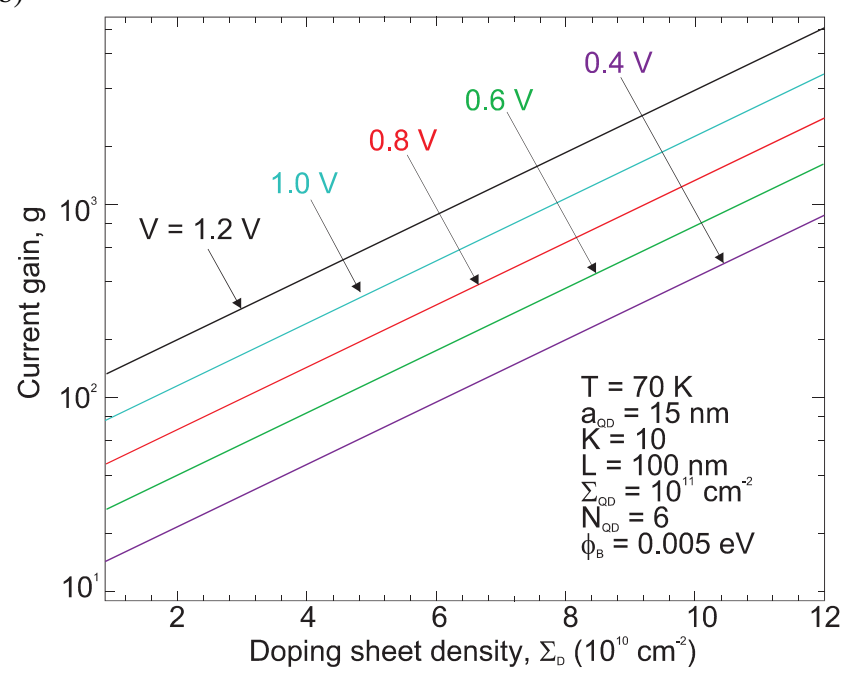

Fig. 8. Current gain versus quantum dot density, $\Sigma_{Q D}$ (a) and doping sheet density, $\Sigma_{D}$ (b) for different bias voltages at temperature $70 \mathrm{~K}$. In theoretical estimation, the following parameters have been assumed: $a_{Q D}=15 \mathrm{~nm}, \Sigma_{Q D}=10^{11} \mathrm{~cm}^{-2}, K=10, L=100 \mathrm{~nm}$, $N_{Q D}=6$, and $\phi_{B}=0.005 \mathrm{eV}$

Additional insight in the current gain gives Fig. 9, where influence of the number of QD layers, $K$, and the spacing between QD layers, $L$, is shown for several bias voltages, assuming typical material parameters of quantum dots. The current gain of the sample containing lower number of quantum dot layers is higher than that of the thicker sample with higher number of QD layers. As it results from Eq. (14), the current gain depends inversely proportionally on the number of quantum dot layers, $K$. The dependence of current gain on the spacing between QD layers, $L$, is stronger in the region of lower values of $L$ [see Fig. 9(b)].

Average carrier number in dots. It is well understood that the carrier number inside the quantum dots is essential to the quantum efficiency and performance of QDIPs. The optimized condition occurs when the ground states are fully oc- 
cupied and the excited states are empty, i.e., two electrons per quantum dot. Then, the optimal dopant density corresponds to twice the quantum dot sheet (areal) density [12]. The dark current increases at higher dopant densities as dopants outnumber, the number of electron states available in the QD ground state. Whereas at reduced dopant densities, there are fewer electrons in the QD ground state for absorption.

a)

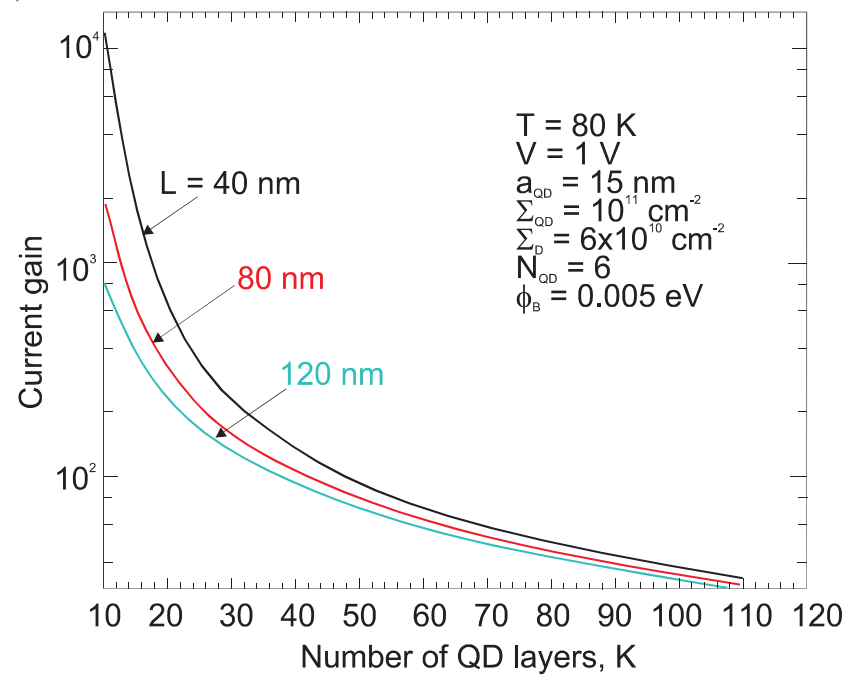

b)

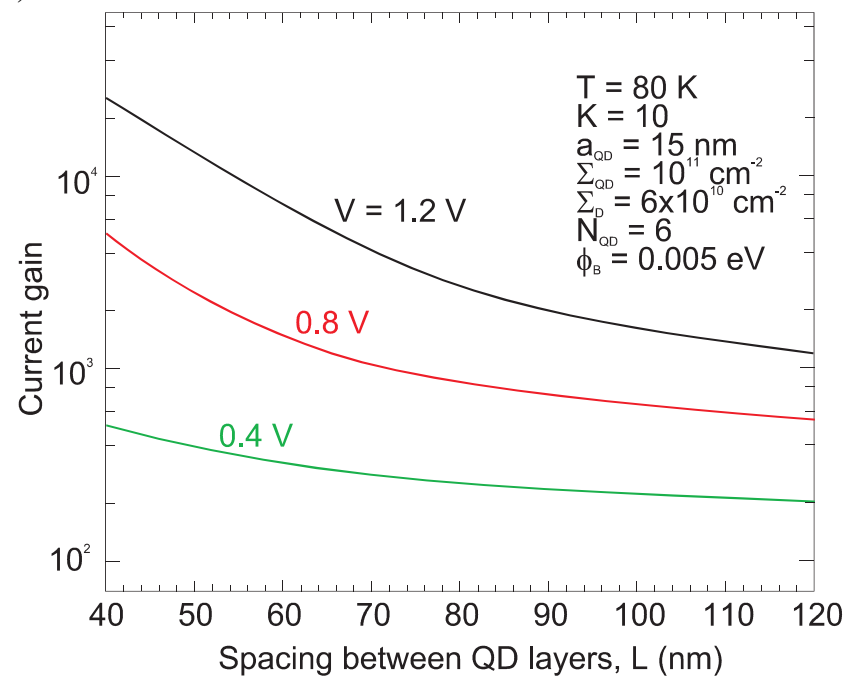

Fig. 9. Current gain as a function of the number of QD layers, $K$ (a) and the spacing between QD layers, $L(\mathrm{~b})$, at operation temperature $80 \mathrm{~K}$. In theoretical estimation, the following parameters have been assumed: $a_{Q D}=15 \mathrm{~nm}, \Sigma_{Q D}=10^{11} \mathrm{~cm}^{-2}, \Sigma_{D}=6 \times 10^{10} \mathrm{~cm}^{-2}$, $N_{Q D}=6$, and $\phi_{B}=0.005 \mathrm{eV}$.

Due to the large size of quantum dots considered in our paper (diameter of $15 \mathrm{~nm}$ and a height of $5 \mathrm{~nm}$ ), the maximum electron number that a QD can accommodate, $N_{Q D}$, is much larger than the average carrier number in the QDs, $\langle N\rangle$. As it is indicated in section 3, the average carrier number can be estimated by solving numerically Eq. (18).

Figure 10 shows the temperature dependence of the estimated average carrier number in the dots at different bias voltages, assuming material parameter marked insight the fig- ure. At a constant bias voltage, increase in dark current with a temperature can be realized through decrease in average number of carriers in the dots.

At constant doping density [see Fig. 10(b); $\Sigma_{D}=5 \times$ $10^{10} \mathrm{~cm}^{-2}$ ], the average number of electrons decreases with increasing QD density and saturates in the range of high $\Sigma_{Q D}$. It can be expected that for lower value of $\Sigma_{Q D}$, in the range below $\Sigma_{D}=5 \times 10^{10} \mathrm{~cm}^{-2}$, the dependence $\langle N\rangle$ on $\Sigma_{Q D}$ is conditioned by carriers from heavy doped detector contact regions.

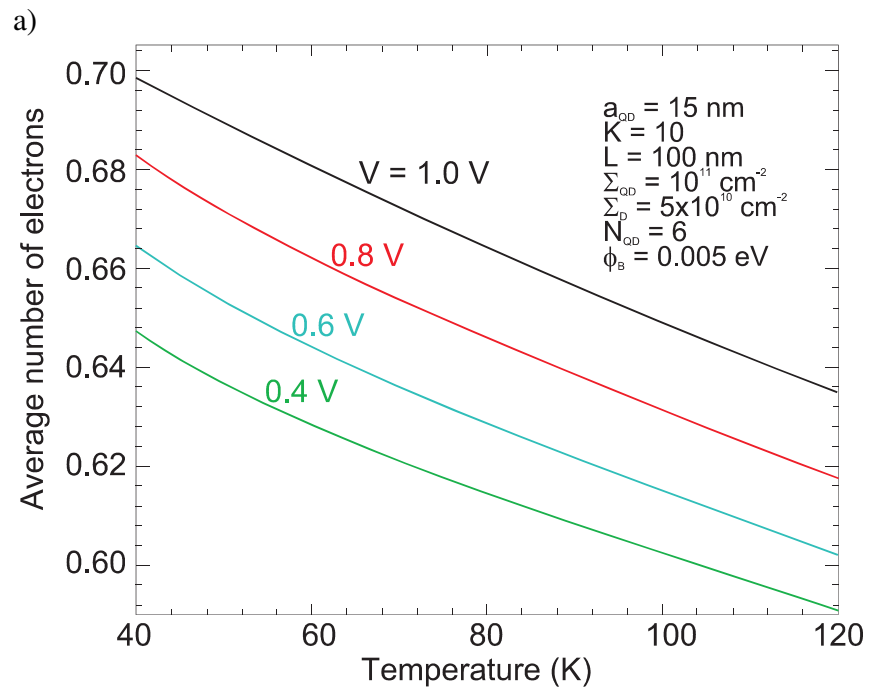

b)

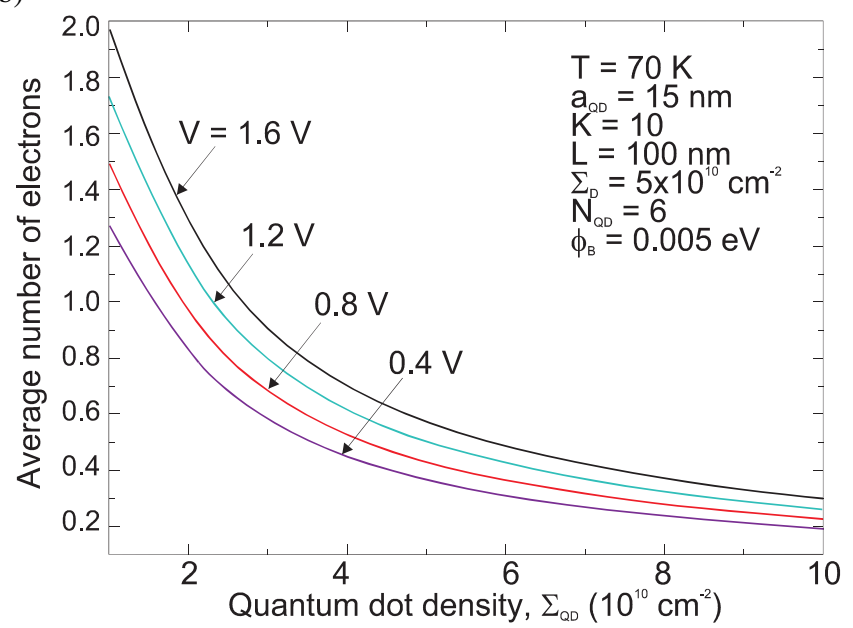

Fig. 10. The calculated average carrier number in one dot as a function of temperature (a) and the quantum dot density, $\Sigma_{Q D}$, (b) for different bias voltages. In theoretical estimation, the following parameters have been assumed: $a_{Q D}=15 \mathrm{~nm}, K=10, L=100 \mathrm{~nm}$,

$$
\Sigma_{D}=5 \times 10^{10} \mathrm{~cm}^{-2}, N_{Q D}=6 \text {, and } \phi_{B}=0.005 \mathrm{eV}
$$

The device structure design of QDIP has also appreciable influence on average number of electrons in quantum dots. Figure 11 presents influence of the number of QD layers, $K$, and the spacing between QD layers, $L$, on $\langle N\rangle$ for several bias voltages assuming typical material parameters of quantum dots. Increasing both $K$ and $L$ causes decreasing average number of carriers due to effective increasing detector's volume. 
a)

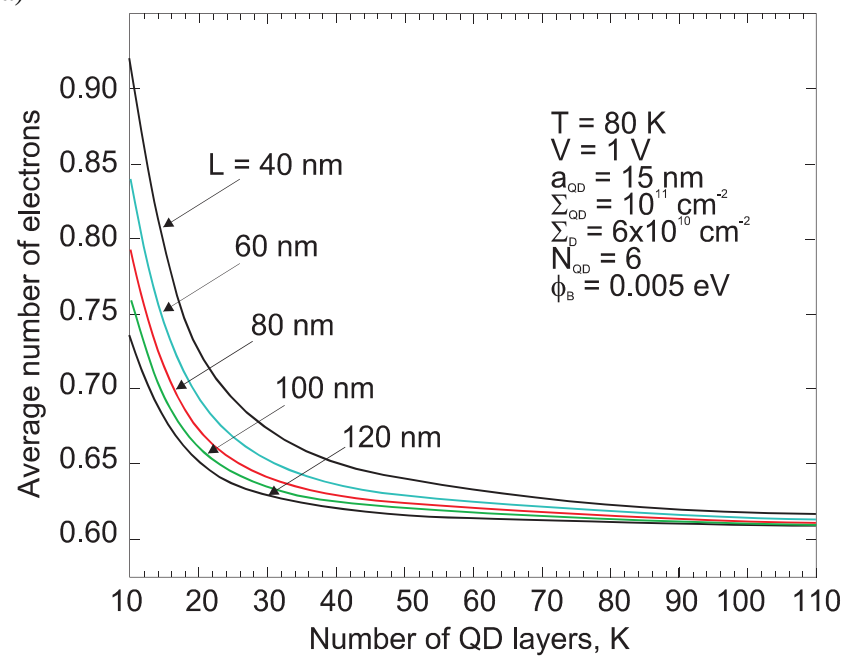

b)

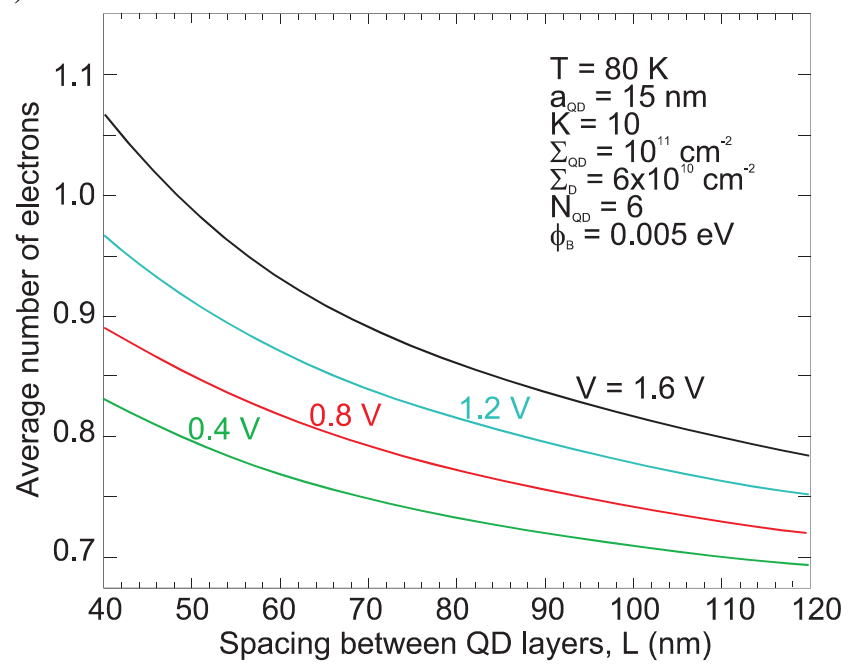

Fig. 11. Average number of electrons as a function of number of QD layers, $K$ (a) and spacing between QD layers, $L$ (b), at operation temperature $80 \mathrm{~K}$. In theoretical estimation, the following parameters have been assumed: $a_{Q D}=15 \mathrm{~nm}, \Sigma_{Q D}=10^{11} \mathrm{~cm}^{-2}$, $\Sigma_{D}=6 \times 10^{10} \mathrm{~cm}^{-2}, N_{Q D}=6$, and $\phi_{B}=0.005 \mathrm{eV}$

4.2. QDIP's characteristics in illuminated conditions. If a detector is under illumination, infrared radiation causes the photoexcitation in quantum dots due to the electron transitions from the bound states in QDs into the continuum states above the inter-QD barriers (see Fig. 1). When the photoexcitation of electrons from QDs dominates their thermionic emission, the photocurrent density can be determined by the following equation

$$
\left\langle j_{\text {photo }}\right\rangle=q \eta \Phi_{s} g
$$

where $\eta$ is the quantum efficiency, $g$ is the photocurrent gain, and $\Phi_{s}$ is the photon flux density incident on a detector.

The absorption coefficient can be estimated using the following equation

$$
\alpha=\frac{K \delta\langle N\rangle \Sigma_{Q D}}{K L}=\frac{\delta\langle N\rangle \Sigma_{Q D}}{L},
$$

where $\delta=2.5 \times 10^{-15} \mathrm{~cm}^{-2}$ is the electron capture cross section coefficient.
Since the quantum efficiency can be approximated by

$$
\eta \approx \alpha t \approx \alpha K L
$$

where $t=K L$, then

$$
\left\langle j_{\text {photo }}\right\rangle=q \Phi_{s} g(\alpha t)=\frac{\delta q\langle N\rangle \Sigma_{Q D} \Phi_{s}}{p_{k}} .
$$

Finally, using equations (3) and (8), we obtain the equation similar to Eq. (18) for the dark conditions

$$
\begin{gathered}
\frac{\delta q \sigma\langle N\rangle \Sigma_{Q D} \Phi_{s}}{p_{k}}+\left\langle j_{\text {dark }}\right\rangle \\
=j_{\max } \frac{\Theta}{\langle N\rangle} \exp \left[q \frac{V+V_{D}-\left(\langle N\rangle / N_{Q D}\right) V_{Q D}}{(K+1) k T}\right],
\end{gathered}
$$

from which we can determine an average number of electrons in QDs $\langle N\rangle$ and next other detector's parameters in illuminated conditions.

Photocurrent and quantum efficiency. As indicates Eq. (19), current gain, and connected with it a number of electrons in quantum dots, has decisive influence in bias voltage dependence of photocurrent. Figure 12 shows that the photocurrent gain is insensibly lower than the current gain. Lower average number of electrons in quantum dots, in comparison with dark conditions, is conditioned by extra photoexcitation of electrons by incident radiation.

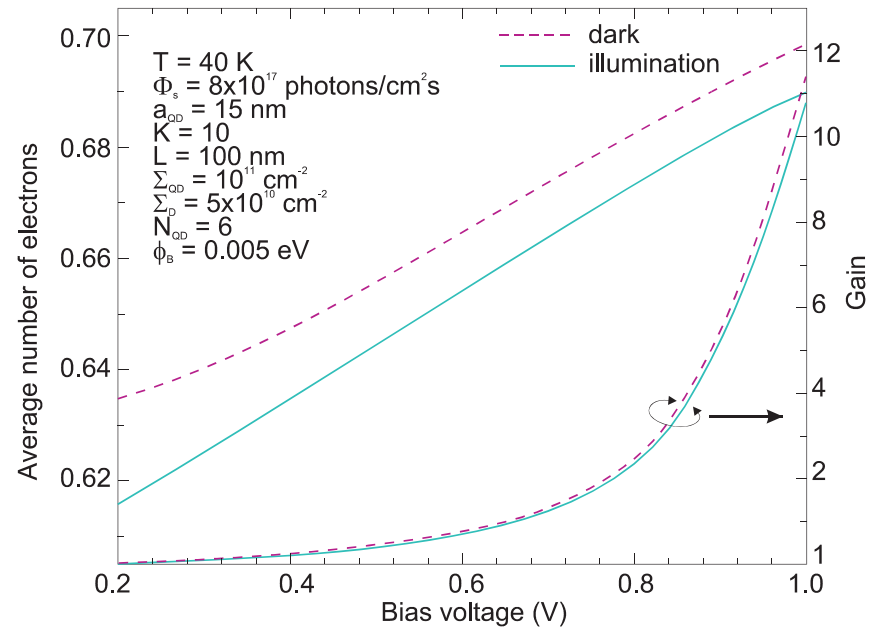

Fig. 12. Dependence of average number of electrons and current gains in dark and illuminated conditions for QDIP detector operating at $40 \mathrm{~K}$. In theoretical estimation, the following parameters have been assumed: $a_{Q D}=15 \mathrm{~nm}, \Sigma_{Q D}=10^{11} \mathrm{~cm}^{-2}$, $\Sigma_{D}=5 \times 10^{10} \mathrm{~cm}^{-2}, N_{Q D}=6, \phi_{B}=0.005 \mathrm{eV}$, and the incident photon flux $\Phi_{s}=8 \times 10^{17}$ photons $/ \mathrm{cm}^{2} \mathrm{~s}$.

In Fig. 3, it is also shown theoretically a predicted curve for QDIP operating at $40 \mathrm{~K}$ in illuminated conditions of incident photon flux density $\Phi_{s}=8 \times 10^{17}$ photons $/ \mathrm{cm}^{2} \mathrm{~s}$. Again, the agreement between the experimental results and theory is good, demonstrating that the elaborated model of QDIP is valid for a wide range of detector operating conditions.

Figure 13 shows the estimated photocurrent as a function of $K$ and $L$. Together with increasing number of the quantum dot layers as well as the spacing between them, the photocurrent decreases. The decisive factor which influences 
the photocurrent is dependence of the electron mobility on an electric field. In the range of $K>30$, a saturation of photocurrent is observed [see Fig. 13(a)]. Also in the range of a low value of bias voltage, the photocurrent saturation is observed [see Fig. 13(b)].

a)

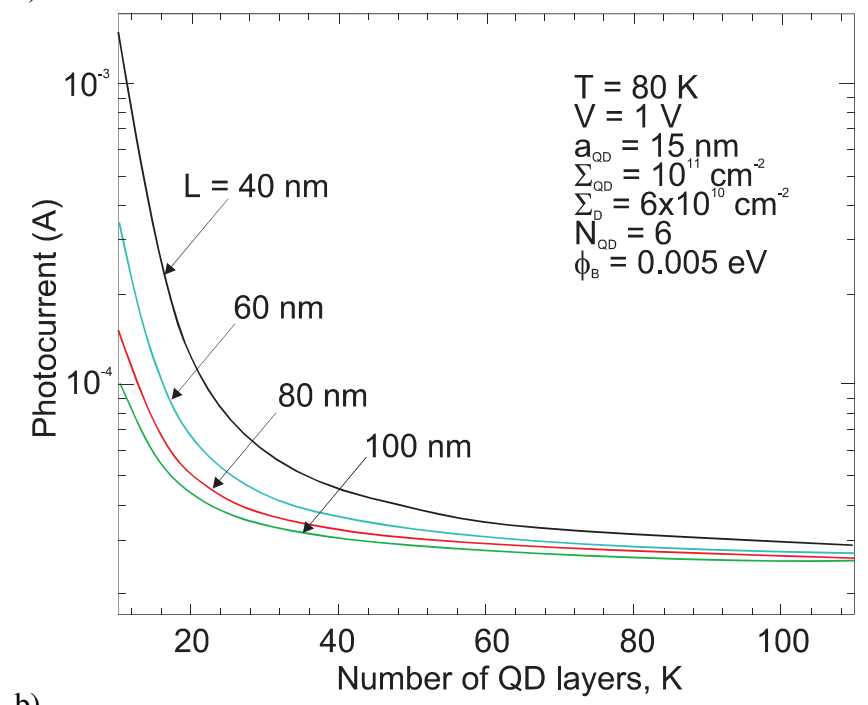

b)

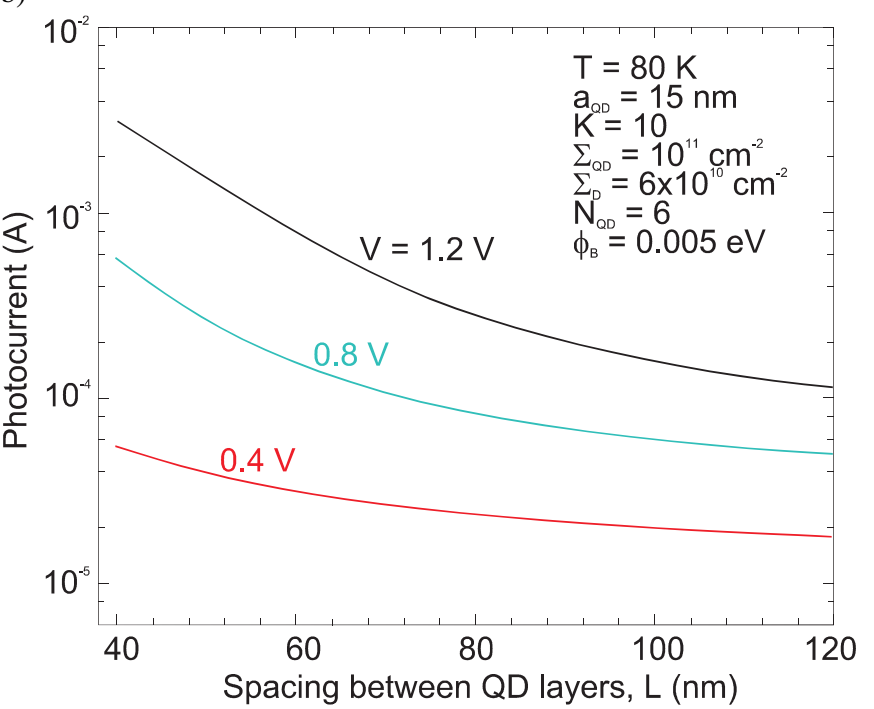

Fig. 13. Photocurrent as a function of the number of QD layers, $K$ (a) and spacing between QD layers, $L$ (b), at operation temperature $80 \mathrm{~K}$. In theoretical estimation, the following parameters have been assumed: $a_{Q D}=15 \mathrm{~nm}, \Sigma_{Q D}=10^{11} \mathrm{~cm}^{-2}, \Sigma_{D}=6 \times 10^{10} \mathrm{~cm}^{-2}$, $N_{Q D}=6$, and $\phi_{B}=0.005 \mathrm{eV}$

It is obvious that the density of quantum dots and doping sheet density have decisive influence on quantum efficiency. Figure 14 shows the change of the quantum efficiency with the number of quantum dot layers, $K$, and with the spacing between them, $L$, at different values of bias voltage. Increasing quantum dot layers results in increasing active volume of a detector and the quantum efficiency increases. It can be noticed, that the quantum efficiency is a weak function of a spacing value between QD layers [see Fig. 12(a)] and bias voltage [see Fig. 12(b)]. a)

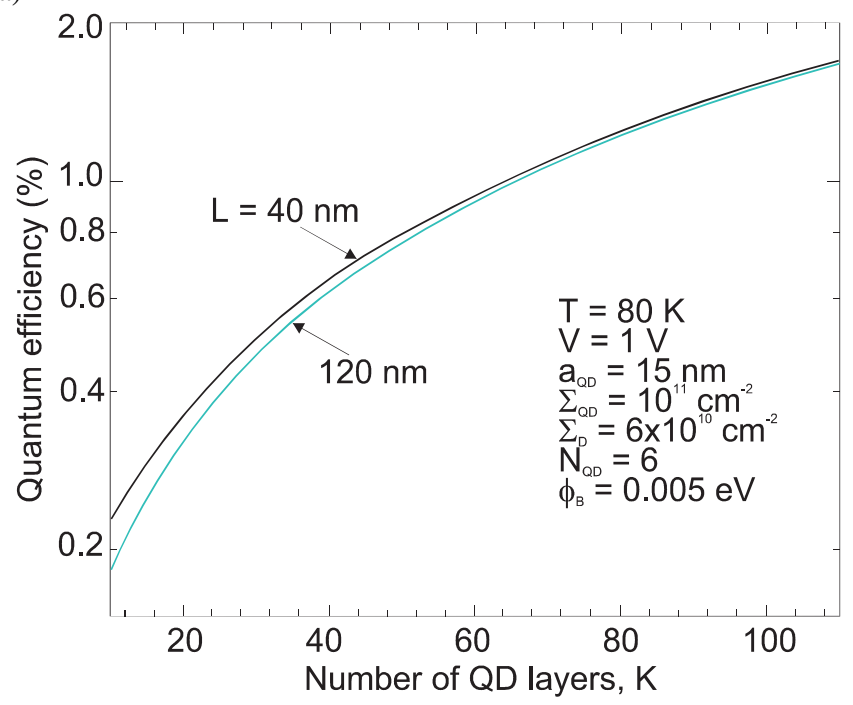

b)

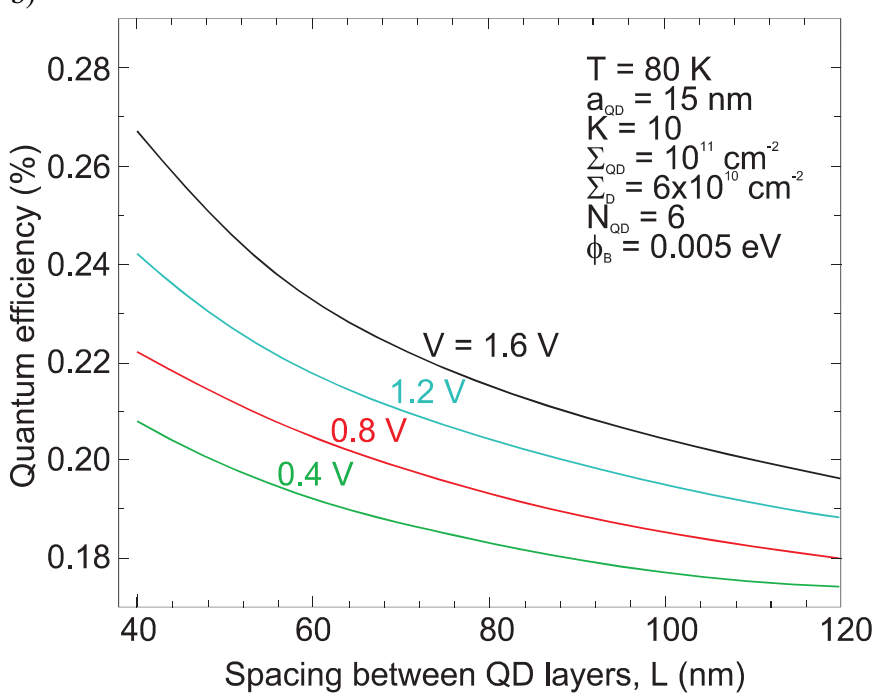

Fig. 14. The dependence of quantum efficiency on the number of QD layers, $K$ (a) and the spacing between QD layers, $L$ (b), at operation temperature $80 \mathrm{~K}$. In theoretical estimation, the following parameters have been assumed: $a_{Q D}=15 \mathrm{~nm}, \Sigma_{Q D}=10^{11} \mathrm{~cm}^{-2}$, $\Sigma_{D}=6 \times 10^{10} \mathrm{~cm}^{-2}, N_{Q D}=6$, and $\phi_{B}=0.005 \mathrm{eV}$

Current responsivity. The responsivity is the ratio of the detector photocurrent to the incident photon power and it can be defined as

$$
R_{i}=\frac{\left\langle j_{\text {photo }}\right\rangle}{h v \Phi_{s}}
$$

In our considerations, we will concentrate on dependence of responsivity on the temperature, $T$, density of the quantum dots, $\Sigma_{Q D}$, and the doping density, $\Sigma_{D}$.

Figure 15(a) shows the change of the responsivity with a temperature at different values of bias voltages. The responsivity increases both with bias voltage as well as with temperature, which are next influenced by a current gain. Also the change of a carrier number inside the QDs plays an important role on the temperature dependence of the responsivity in QDIPs. The higher the dark current is, the more the charge 
inside the QDs will be. This feature enhances the responsivity and the performance of the QDIPs at higher temperature.

a)

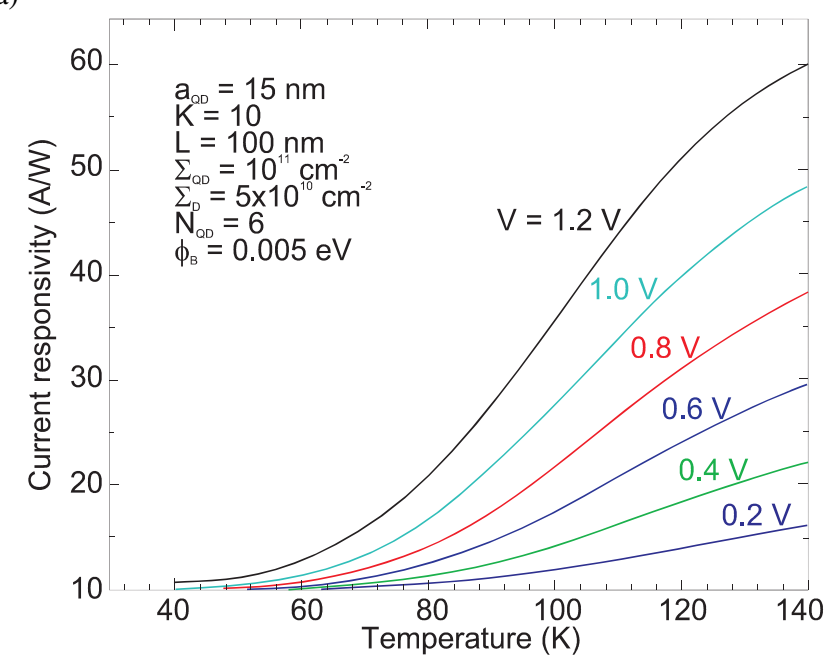

b)

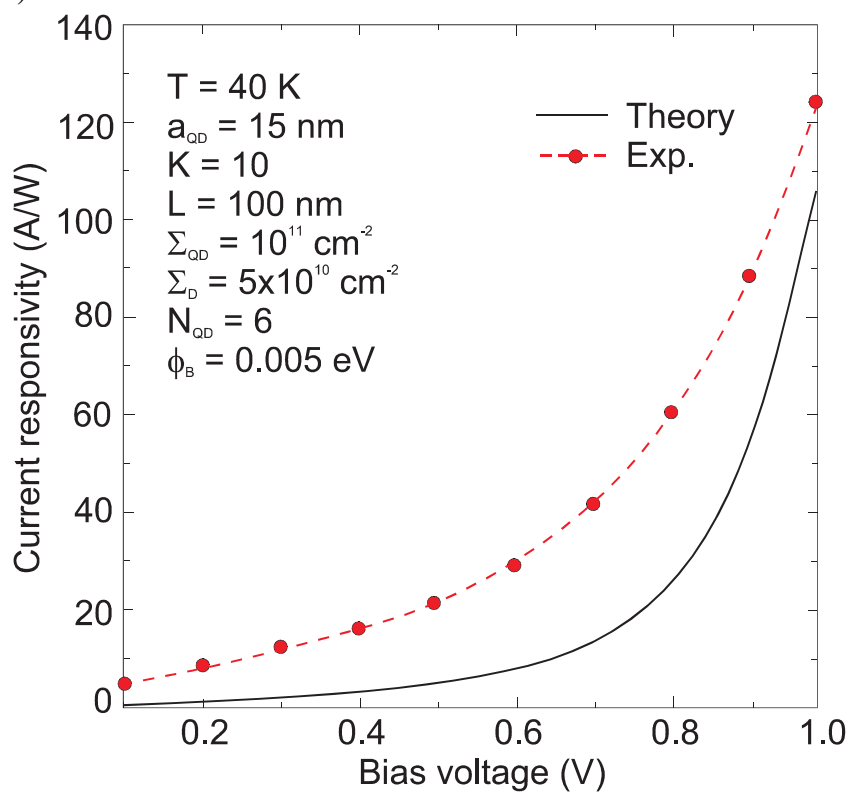

Fig. 15. Current responsivity versus temperature at different applied biasing voltages (a) and versus bias voltage at $40 \mathrm{~K}$. The experimental data at $40 \mathrm{~K}$ are after Ref. 16. In theoretical estimation, the following parameters have been assumed: $a_{Q D}=15 \mathrm{~nm}, \Sigma_{Q D}=10^{11} \mathrm{~cm}^{-2}$, $\Sigma_{D}=5 \times 10^{10} \mathrm{~cm}^{-2}, K=10, L=100 \mathrm{~nm}, N_{Q D}=6$, $\phi_{B}=0.005 \mathrm{eV}$, and $\Phi_{s}=8 \times 10^{17}$ photons $/ \mathrm{cm}^{2} \mathrm{~s}$

It can be expected that the saturation of responsivity with the increasing $V$ and $T$ results from the changes of electron mobility (see Table 1). According to Ref. 15, the current gain in the range of the higher bias voltages and temperature can even decrease. The mechanism that can make the gain go down with bias is a decrease in the drift velocity due to shallow trap limited mobility. So, the photocurrent and responsivity depend on the trap energy, $E_{t}$. We notice that the obtained calculated results give good agreement with experimental work.

Increasing quantum dot density, at constant doping level, results in decreasing the current responsivity due to decreas- ing current gain [Fig. 16(a)]. The highest changes of responsivity occur in the range of low quantum dot density. At higher densities, the responsivity saturates independently on biasing. In turn, increasing doping density causes increase in current responsivity because the number of electrons in QDs increases (also current gain increases) [see Fig. 16(b)].

a)

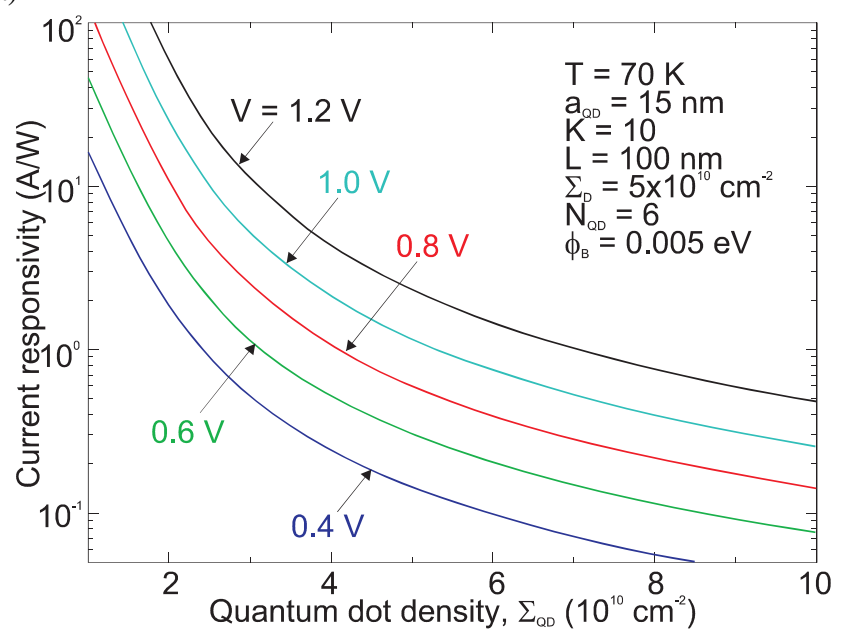

b)

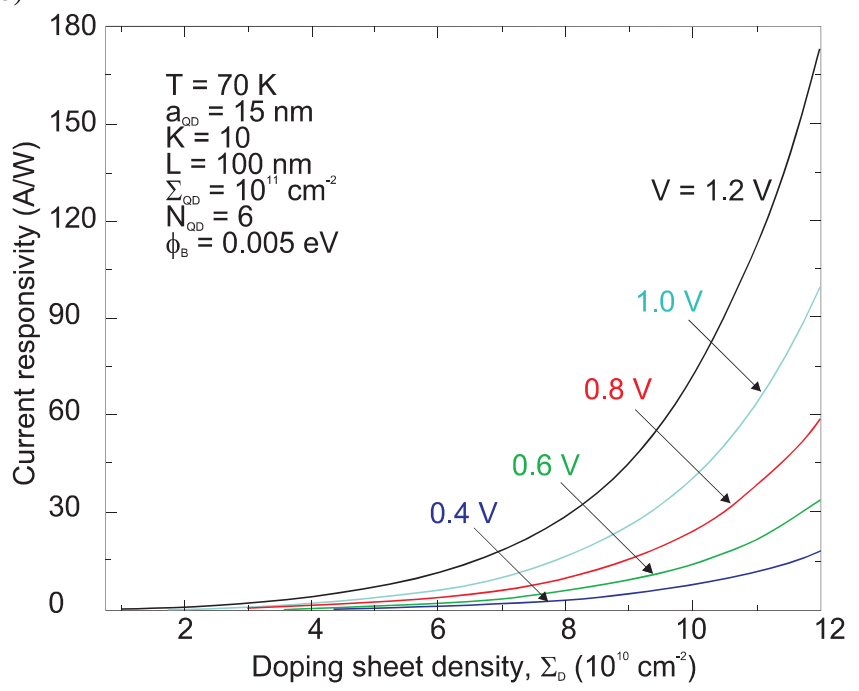

Fig. 16. Current responsivity versus the quantum dot density, $\Sigma_{Q D}$ (a) and the doping sheet density, $\Sigma_{D}$ (b) for different bias voltages at temperature $70 \mathrm{~K}$. In theoretical estimation, the following parameters have been assumed: $a_{Q D}=15 \mathrm{~nm}, K=10, L=100 \mathrm{~nm}$, $N_{Q D}=6$, and $\phi_{B}=0.005 \mathrm{eV}$

Detectivity. It results from considerations presented in section 4.2 that the current gain, in dark and illuminated conditions, is similar (see Fig. 12). Taking into account that thermally limited detectivity is equal to

and

$$
D^{*}=\frac{R_{i} \sqrt{A}}{\left(4 q I_{\text {dark }} g\right)^{1 / 2}},
$$

$$
\begin{gathered}
I_{\text {dark }}=q \Sigma_{Q D} K\left(G_{\text {th }}+G_{\text {tun }}\right) g A, \\
I_{\text {photo }}=K \delta q\langle N\rangle \Sigma_{Q D} \Phi_{s} g A,
\end{gathered}
$$

the following equation for detectivity can be obtained 


$$
D^{*}=\frac{\delta \sqrt{\Sigma_{Q D} K}\langle N\rangle}{2 q h \nu\left\{\exp \frac{\pi \eta^{2}\langle N\rangle}{m^{*} k T a_{Q D}^{2}}\left[G_{o} \exp \left(-\frac{\varepsilon_{Q D}}{k T}\right)+G_{o t} \exp \left(-\frac{4}{3} \frac{\sqrt{2 m^{*} q}}{\eta} \frac{\phi_{B}^{3 / 2}}{E}\right) \exp \left(-\frac{\Delta \varepsilon}{k T}\right)\right]\right\}^{1 / 2}}
$$

Figures 17-19 illustrate the change of detectivity with different parameters: temperature, applied bias voltage, doping sheet density, number of quantum dot layers, and spacing between quantum dot layers. In calculations, the typical values of parameters given previously have been assumed.

a)

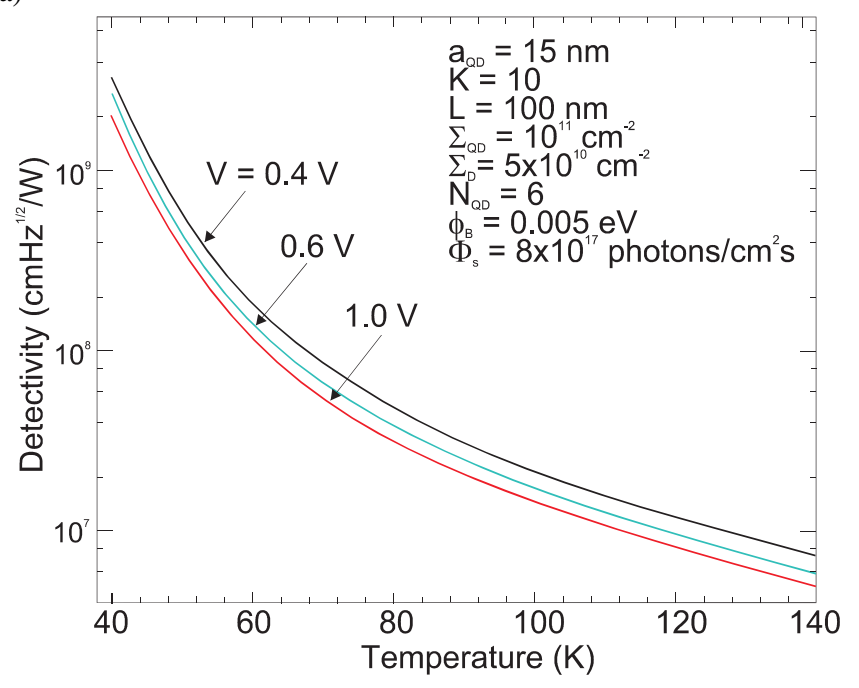

b)

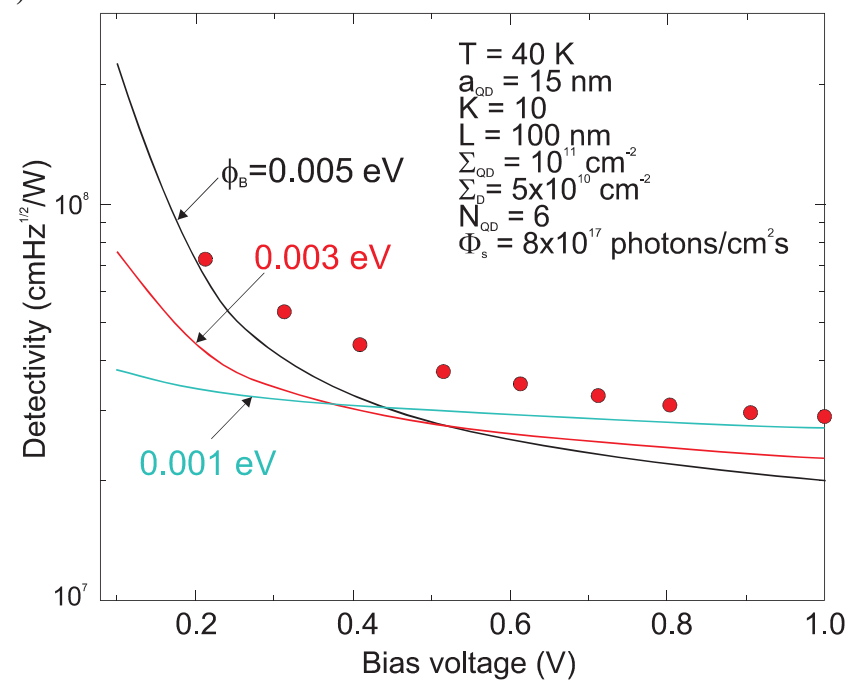

Fig. 17. Detectivity as a function of temperature (a) and bias voltage (b). In theoretical estimation, the following parameters have been assumed: $a_{Q D}=15 \mathrm{~nm}, \Sigma_{Q D}=10^{11} \mathrm{~cm}^{-2}, \Sigma_{D}=5 \times 10^{10} \mathrm{~cm}^{-2}$, $K=10, L=100 \mathrm{~nm}, N_{Q D}=6$, and $\phi_{B}=0.005 \mathrm{eV}$. The experimental data at $40 \mathrm{~K}$ are after Ref. 16

As expected, a rapid decrease in detectivity occurs with the increase in a temperature due to contribution of thermal generation [Fig. 17(a)]. Low value of detectivity results from small quantum efficiency, typically $0.2-0.3 \%$ [see Fig. 14(b)]. Changes of detectivity in dependence on a bias voltage are small. However, the potential barrier, $\phi_{B}$, has stronger influence on detectivity in the region of a low bias voltage below $0.5 \mathrm{~V}$. Increasing barrier height causes increasing detectivity [see Fig. 17(b)]. This effect is also connected with increasing number of electrons occupied quantum dots. Experimental data shown in Fig. 17(b) demonstrate good agreement with theory over wide range of bias voltages.

a)

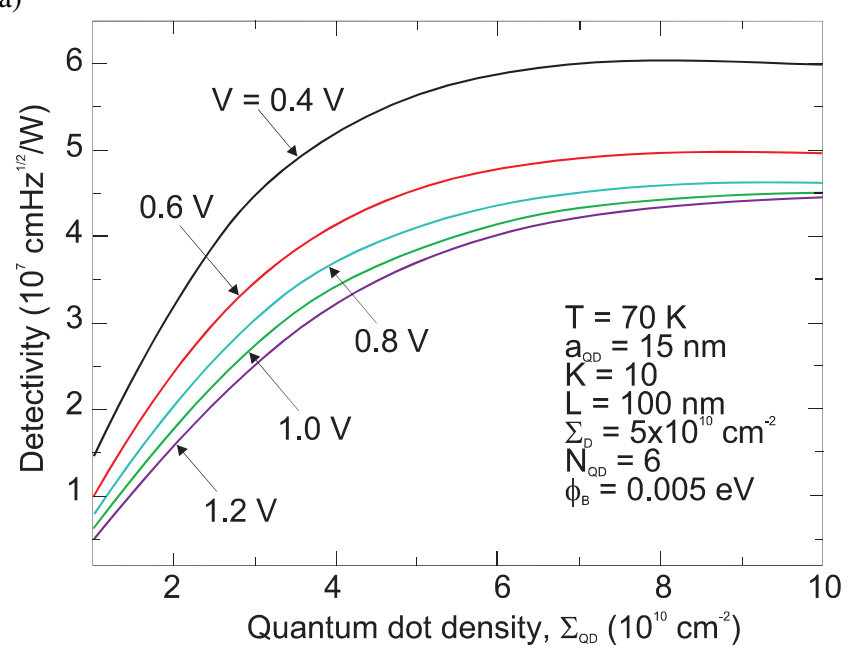

b)

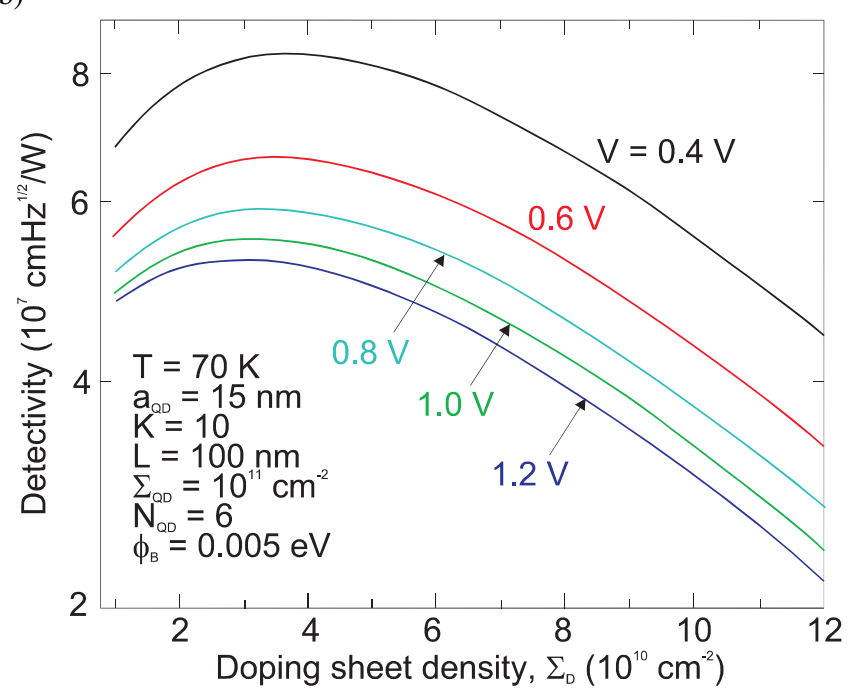

Fig. 18. Detectivity versus the quantum dot density, $\Sigma_{Q D}$ (a) and the doping sheet density, $\Sigma_{D}$ (b) for different bias voltages at temperature $70 \mathrm{~K}$. In theoretical estimation, the following parameters have been assumed: $a_{Q D}=15 \mathrm{~nm}, K=10, L=100 \mathrm{~nm}, N_{Q D}=6$, and $\phi_{B}=0.005 \mathrm{eV}$

It is obvious that increasing quantum dot density results in increasing detectivity [Fig. 18(a)]. In this way, both the active 
detector's area and the absorption coefficient increase. However, with increasing density of QDs, the detectivity saturates since the number of electrons in QDs effectively decreases.

In the same way affect changes of doping sheet density on detectivity [see Fig. 18(b)]. At the beginning, increasing doping density results in increasing detectivity. In this range of doping density, influence of increasing number of carriers in QDs prevails influence of thermal generation. However, in the range of higher doping density (above $3.5 \times 10^{10} \mathrm{~cm}^{-2}$ for bias voltage $0.4 \mathrm{~V}$ ) influence of thermal generation is dominant and detectivity decreases. With increasing bias voltage, the maximum of detectivity moves in a direction of lower doping concentration.

a)

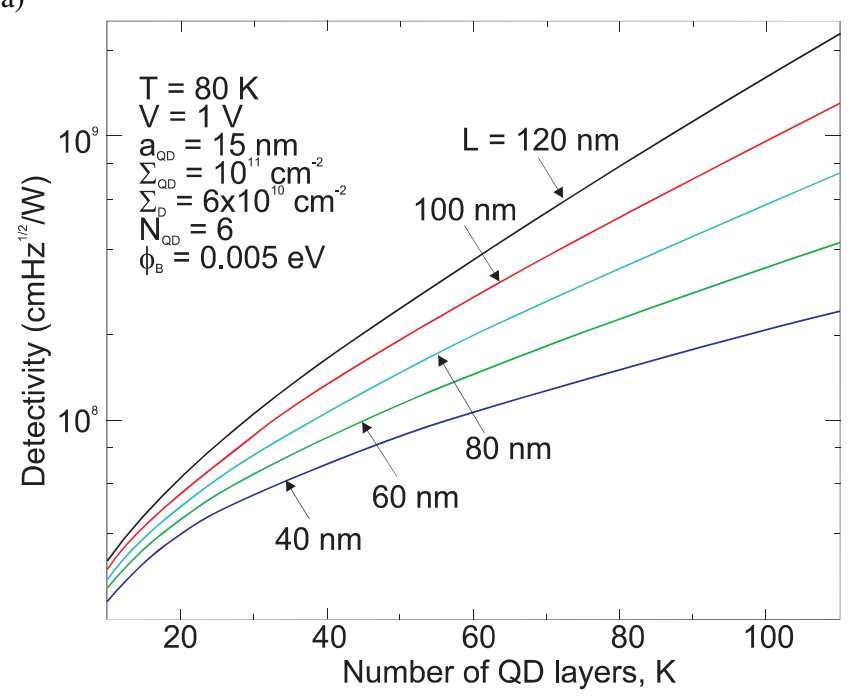

b)

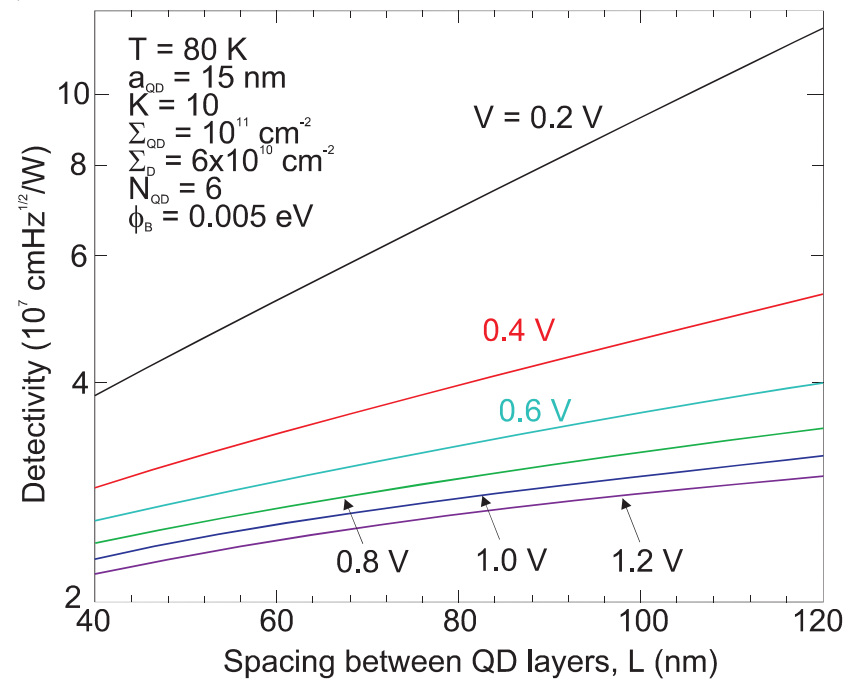

Fig. 19. The dependence of detectivity on the number of QD layers, $K$ (a) and the spacing between QD layers, $L(\mathrm{~b})$, at operation temperature $80 \mathrm{~K}$. In theoretical estimation, the following parameters have been assumed: $a_{Q D}=15 \mathrm{~nm}, K=10, \Sigma_{Q D}=10^{11} \mathrm{~cm}^{-2}$, $\Sigma_{D}=6 \times 10^{10} \mathrm{~cm}^{-2}, N_{Q D}=6$, and $\phi_{B}=0.005 \mathrm{eV}$

Detectivity of QDIPs depends essentially also on the detector design. Figure 19 presents the change of the detectivity with the number of the quantum dot layers, $K$, and with the spacing between, $L$, at different values of bias voltage. Increasing number of quantum dot layers results in increasing active volume of a detector and the detectivity increases. Figure 19(b) indicates that the detectivity is a weak function of a spacing value between QD layers especially at higher bias voltages.

\section{Conclusions}

The intention of this paper is theoretical evaluation of QDIP performance under dark and illumination conditions in dependence on different values of material parameters and design of active detector's region. The developed algorithm is based on a model, previously published by Ryzhii and co-workers, and assumes that both thermionic emission and field-assisted tunnelling mechanisms determine the dark current of quantum dot detectors. The model permits to calculate the dark current, current gain, average number of electrons in quantum dots, photocurrent, detector responsivity and detectivity as a function of the structural parameters. Moreover, it explains some features of QDIP characteristics.

The obtained theoretical predictions explain a sharp dark current-voltage characteristic of QDIPs and strong dependence of the dark current on the density of quantum dots in the detector and the doping level of the active region observed in experiments.

In general, however, it is rather difficult to compare theoretically the predicted performance of QDIPs with experimental data. Such comparison requires accurate control of quantum dot detector's design parameters. In the most of published papers, the required parameters are not given. On account of this, only part of the predicted detector characteristics are compared with these measured experimentally. Good agreement has been obtained between theoretical predictions and experimental data published in Ref. 16 (dark current and photocurrent, current gain, current responsivity and detectivity).

Improving QD uniformity is a key issue in the increasing absorption coefficient and improving detector performance. Optimization of the QDIP architecture is still an open area. Since some of the design parameters depend on a device structure (photoconductive and noise gains, dark current, quantum efficiency), the performance is still being improved. This paper gives insight on the choice of a way to improve performance of quantum dot infrared detectors.

Acknowledgements. This paper has been done under financial support of the Polish Ministry of Sciences and Higher Education, Key Project POIG.01.03.01-14-016/08 "New Photonic Materials and their Advanced Application".

\section{REFERENCES}

[1] S.D. Gunapala and S.V. Bandara, "GaAs/AlGaAs based quantum well infrared photodetector focal plane arrays", in Handbook of Infrared Detection Technologies, edited by M. Henini and M. Razeghi, pp. 83-119, Elsevier, Oxford, 2002.

[2] A. Rogalski, "Quantum well photoconductors in infrared detector technology", J. Appl. Phys. 93, 4355-4391 (2003). 
[3] E. Towe and D. Pan, "Semiconductor quantum-dot nanostructures: Their application in a new class of infrared photodetectors", IEEE J. Sel. Top. Quant. 6, 408-421 (2000).

[4] P. Boucaud and S. Sauvage, "Infrared photodetection with semiconductor self-assembled quantum dots", C.R. Phys. 4, 1133-1154 (2003).

[5] P. Bhattacharya, A.D. Stiff-Roberts, and S. Chakrabarti, "Midinfrared quantum dot photoconductors", in Mid-infrared Semiconductor Optoelectronics, pp. 487-513, edited by A. Krier, Springer Verlag, 2007.

[6] S. Krishna, S.D. Gunapala, S.V. Bandara, C. Hill, and D.Z. Ting, "Quantum dot based infrared focal plane arrays", Proc. IEEE 95, 1838-1852 (2007).

[7] E. Varley, M. Lenz, S.J. Lee, J.S. Brown, D.A. Ramirez, A. Stintz, and S. Krishna, "Single bump, two-color quantum dot camera", Appl. Phys. Lett. 91, 081120 (2007).

[8] V. Ryzhii, I. Khmyrova, V. Pipa, V. Mitin, and M. Willander, "Device model for quantum dot infrared photodetectors and their dark-current characteristics", Semicond. Sci. Tech. 16, 331-338 (2001).

[9] V. Ryzhii, I. Khmyrova, V. Mitin, M. Stroscio, and M. Willander, "On the detectivity of quantum-dot infrared photodetectors", Appl. Phys. Lett. 78, 3523-3525 (2001).

[10] V. Ryzhii, "Analysis of the photocurrent in quantum dot infrared photodetectors", Jpn. J. Appl. Phys. 40, L148-L150 (2001).

[11] I. Vurgaftman, Y. Lam, and J. Singh, "Carrier thermalization in sub-three-dimensional electronic systems: Fundamental limits on modulation bandwidth in semiconductor lasers", Phys. Rev. B50, 14309-14326 (1994).
[12] J. Phillips, "Evaluation of the fundamental properties of quantum dot infrared detectors", J. Appl. Phys. 91, 4590-4594 (2002).

[13] A.D. Stiff-Roberts, "Contrubution of field-assisted tunneling emission to dark current in InAs-GaAs quantum dot infrared photodetectors", IEEE Photonic. Techn. L. 16, 867-869 (2004).

[14] G.M. Williams, R.E. DeWames, C.W. Farley, and R.J. Anderson, "Excess tunnel currents in AlGaAs/GaAs multiple quantum well infrared detectors", Appl. Phys. Lett. 60, 1324-1326 (1992).

[15] H. Lim, B. Movaghar, S. Tsao, M. Taguchi, W. Zhang, A.A. Quivy, and M. Razeghi, "Gain and recombination dynamics of quantum-dot infrared photodetectors", Phys. Rev. B74, 205321 (2006).

[16] J. Philips, P. Bhattacharya, S.W. Kennerly, D.W. Beekman, and M. Duta, "Self-assembled InAs-GaAs quantum dot intersubband detectors", IEEE J. Quantum Elect. 35, 936-943 (1999).

[17] J. Singh, Electronic and Optoelectronic Properties of Semiconductor Structures, Cambridge University Press, New York, 2003.

[18] Z. Ye, J.C. Campbell, Z. Chen, E.-T. Kim, and A. Madhukar, "Noise and photoconductive gain in InAs quantum-dot infrared photodetectors", Appl. Phys. Lett. 83, 1234-1236 (2003).

[19] S.Y. Wang, M.C. Lo, H.Y. Hsiao, H.S. Ling, and C.P. Lee, "Temperature dependent responsivity of quantum dot infrared photodetectors", Infrared Phys. Techn. 50, 166-170 (2007).

[20] J.C. Campbell and A. Madhukar, "Quantum-dot infrared photodetectors", Proc. IEEE 95, 1815-1827 (2007). 